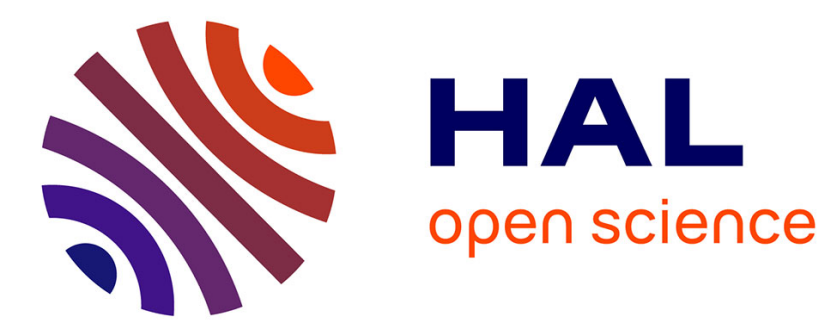

\title{
HLLC-type Riemann solver with approximated two-phase contact for the computation of the Baer-Nunziato two-fluid model
}

Hippolyte Lochon, Frédéric Daude, Pascal Galon, Jean-Marc Hérard

\section{- To cite this version:}

Hippolyte Lochon, Frédéric Daude, Pascal Galon, Jean-Marc Hérard. HLLC-type Riemann solver with approximated two-phase contact for the computation of the Baer-Nunziato two-fluid model. Journal of Computational Physics, 2016, 10.1016/j.jcp.2016.09.015 . hal-01367268

\section{HAL Id: hal-01367268 \\ https://hal.science/hal-01367268}

Submitted on 15 Sep 2016

HAL is a multi-disciplinary open access archive for the deposit and dissemination of scientific research documents, whether they are published or not. The documents may come from teaching and research institutions in France or abroad, or from public or private research centers.
L'archive ouverte pluridisciplinaire HAL, est destinée au dépôt et à la diffusion de documents scientifiques de niveau recherche, publiés ou non, émanant des établissements d'enseignement et de recherche français ou étrangers, des laboratoires publics ou privés. 


\title{
HLLC-type Riemann solver with approximated two-phase contact for the computation of the Baer-Nunziato two-fluid model
}

\author{
H. Lochon ${ }^{\mathrm{a}, \mathrm{b}, \mathrm{e}}$, F. Daude ${ }^{\mathrm{a}, \mathrm{b}}$, P. Galon ${ }^{\mathrm{b}, \mathrm{c}}$, and J.-M. Hérard ${ }^{\mathrm{d}, \mathrm{e}}$ \\ ${ }^{a}$ EDF R\&D, Département AMA, 7 Boulevard Gaspard Monge, 91120 Palaiseau, France \\ bIMSIA, UMR EDF/CNRS/CEA/ENSTA 9219, Université Paris-Saclay, 828 Boulevard des Maréchaux, \\ 91762 Palaiseau Cedex, France \\ ${ }^{\mathrm{C}}$ CEA, DEN, DANS, DM2S, SEMT, DYN, 91191 Gif-sur-Yvette Cedex, France \\ dEDF R\&D, Département MFEE, 6 Quai Watier, 78401 Chatou Cedex, France \\ eI2M, UMR CNRS 7373, Technopôle Château-Gombert, 39 rue F. Joliot Curie, 13453 Marseille Cedex 13, France
}

\begin{abstract}
The computation of compressible two-phase flows with the Baer-Nunziato model is addressed. Only the convective part of the model that exhibits non-conservative products is considered and the source terms of the model that represent the exchange between phases are neglected. Based on the solver proposed by Tokareva \& Toro 42, a new HLLC-type Riemann solver is built. The key idea of this new solver lies in an approximation of the two-phase contact discontinuity of the model. Thus the Riemann invariants of the wave are approximated in the "subsonic" case. A major consequence of this approximation is that the resulting solver can deal with any Equation of State. It also allows to bypass the resolution of a non-linear equation based on those Riemann invariants. We assess the solver and compare it with others on 1D Riemann problems including grid convergence and efficiency studies. The ability of the proposed solver to deal with complex Equations Of State is also investigated. Finally, the different solvers have been compared on challenging 2D test-cases due to the presence of both material interfaces and shock waves: a shock-bubble interaction and underwater explosions. When compared with others, the present solver appears to be accurate, efficient and robust.
\end{abstract}

\section{Introduction}

Different strategies are used to model compressible two-phase flows. Unlike the homogeneous approaches, the two-fluid approaches do not consider a mixture of the two phases with supposed equilibrium between phases. The two-fluid models are built using an averaging procedure of the local conservation laws [30, 17. If no phasic equilibrium is assumed, two-pressure two-fluid models are obtained. As shown by Ransom and Hicks [39, who proposed one of the first two-pressure two-fluid models, this class of models exhibits pleasant mathematical properties, particularly hyperbolic systems. More recently, Baer and Nunziato [5] proposed another model of this class to study granular explosive materials. Then, the model has been used in a wider range of applications and many different closure laws have been proposed to that purpose [23, 40, 22, 8, 21. The mathematical properties of the Baer-Nunziato model without any source term were first studied by Embid and Baer [19. The main difficulty of the convective part of the model lies in the fact that it cannot be written under a conservative form. However, a detailed analysis of the system shows that the non-conservative products are associated with a linearly degenerate characteristic field due to the particular choice of closure laws of the Baer-Nunziato model. Thus the non-conservative products are not active across shock waves resulting in unique jump conditions. 
Many Finite Volume schemes have been proposed to tackle the numerical resolution of the BaerNunziato model, or similar forms, with different treatments of the non-conservative terms. Saurel and Abgrall [40] proposed to use a "free streaming" condition to discretize the non-conservative terms together with Rusanov and HLL schemes. Complete Riemann solvers that take into account all the characteristic waves of the model have then been proposed. Schwendeman et al. [41 and later Deledicque and Papalexandris [16] built Godunov-type schemes to that purpose. For the treatment of the non-conservative terms, Schwendeman et al. 41 proposed the "thin layer approximation": the associated linearly degenerate wave is approximated by a thin layer across which the solution is assumed to be smooth. This approximation has also been used in the HLLC-type solver proposed by Tokareva and Toro [42. Simpler but incomplete Riemann solvers have been proposed based on HLLtype solver [3] and HLLC-type solvers [34, 20]. The so-called VFRoe-ncv scheme used by Gallouët et al. 21] is another attempt to tackle the non-conservative framework. Extension of the Osher scheme to non-conservative systems has also been applied to the Baer-Nunziato model [18. Finally, relaxation approaches have been applied to both the isentropic and the complete Baer-Nunziato model [2, 9, 10,

In this paper, we propose a new approximation of the linearly degenerate wave associated with the non-conservative terms. This approximation is based on a physically-relevant simplification of the Riemann invariants of the corresponding wave in the "subsonic" case. We propose to use it in a HLLCtype Riemann solver based on the work of Tokareva and Toro [42. It results in a complete Riemann solver that can deal with any Equation Of State in a straightforward way thanks to the proposed approximation. Another important feature of the present solver is that no iterative method to solve a non-linear equation is required unlike the original HLLC-type solver. Indeed, the intermediate states of the Riemann problem are approximated as a solution of a linear system. Regarding the integration of the non-conservative terms, our new approximation is in agreement with the "thin layer approximation", therefore we treat them as in [41, 42. We compare this new solver with the Rusanov scheme and the HLLC-type solver [42] on both 1D and 2D test-cases. A detailed analysis of the accuracy of the solver is carried out, based on several grid convergence studies on 1D Riemann problems. We also pay a particular attention to the case of vanishing phases as it is involved in a lot of practical configurations and may raise robustness problems. The proposed scheme provides similar results as the original HLLC-type solver in most numerical test-cases. Moreover it appears to be very robust and computationally efficient.

Thus the paper is organized as follows. Section 2 introduces the governing equations of the BaerNunziato model. We also recall the main mathematical properties of the model in order to describe the general solution of the Riemann problem, particularly the two subsonic configurations. Section 3 is devoted to the numerical scheme. We first present the approximation of the two-phase contact which is the keystone of the scheme. Then we detailed the HLLC-type Riemann solver based on this approximation and we provide the complete numerical flux for the associated Finite Volume scheme. In section 4, we assess the proposed numerical scheme. Seven 1D Riemann problems have been carefully chosen to compare it with the Rusanov scheme and the HLLC-type scheme proposed by Tokareva and Toro [42. Grid convergence studies are carried out on half of the test-cases in order to provide a deeper analysis. Besides, we also assess the ability of the solver to deal with complex Equations of State. Finally, three 2D test-cases are studied: a shock-bubble interaction and two underwater explosions. They both include material interfaces and shock waves (with very high pressure differences in the case of underwater explosions) so they are particularly suited to investigate both the accuracy and the robustness of the schemes. 


\section{The Baer-Nunziato model}

\subsection{Governing equations}

The governing equations of the Baer-Nunziato model $[5$ without source terms are the following (when restricting to the one-dimensional case):

$$
\left\{\begin{array}{lll}
\partial_{t}\left(\alpha_{1}\right) & +u_{I} \partial_{x}\left(\alpha_{1}\right) & =0 \\
\partial_{t}\left(\alpha_{k} \rho_{k}\right) & +\partial_{x}\left(\alpha_{k} \rho_{k} u_{k}\right) & =0 \\
\partial_{t}\left(\alpha_{k} \rho_{k} u_{k}\right) & +\partial_{x}\left(\alpha_{k} \rho_{k} u_{k}^{2}+\alpha_{k} p_{k}\right)+(-1)^{k} p_{I} \partial_{x}\left(\alpha_{1}\right) & =0 \\
\partial_{t}\left(\alpha_{k} \rho_{k} e_{k}\right) & +\partial_{x}\left(\alpha_{k} \rho_{k} e_{k} u_{k}+\alpha_{k} p_{k} u_{k}\right)+(-1)^{k} p_{I} u_{I} \partial_{x}\left(\alpha_{1}\right) & =0
\end{array}\right.
$$

The subscript $k$ is the index of the phase $(k=1,2)$. Thus, $\alpha_{k}$ which is the volume fraction of phase $k$, lies in $] 0,1[$ and complies with the following relation:

$$
\alpha_{1}+\alpha_{2}=1
$$

$\rho_{k}, u_{k}$ and $p_{k}$ respectively refer to the mean density, the mean velocity and the mean pressure of phase $k$. The specific total energy $e_{k}$ is given by:

$$
e_{k}=\varepsilon_{k}+\frac{1}{2} u_{k}^{2}
$$

where $\varepsilon_{k}$ is the specific internal energy of phase $k$, linked to the density and pressure by an Equation Of State (EOS):

$$
\varepsilon_{k}=\varepsilon_{k}\left(\rho_{k}, p_{k}\right)
$$

Thanks to the EOS, the celerity $c_{k}$ of acoustics waves in phase $k$ is defined using the following:

$$
\rho_{k} c_{k}^{2}=\left(\partial_{p_{k}} \varepsilon_{k}\right)^{-1}\left(\frac{p_{k}}{\rho_{k}}-\rho_{k}\left(\partial_{\rho_{k}} \varepsilon_{k}\right)\right)
$$

The specific entropy $s_{k}$ of phase $k$ complies with:

$$
c_{k}^{2}\left(\partial_{p_{k}} s_{k}\right)+\left(\partial_{\rho_{k}} s_{k}\right)=0
$$

and the temperature $T_{k}$ is defined as:

$$
\frac{1}{T_{k}}=\left(\partial_{p_{k}} \varepsilon_{k}\right)^{-1}\left(\partial_{p_{k}} s_{k}\right)
$$

The closure laws for the interfacial velocity and pressure $\left(u_{I}, p_{I}\right)$ used by Baer and Nunziato [5] are the following:

$$
\left(u_{I}, p_{I}\right)=\left(u_{2}, p_{1}\right)
$$

Many other closure laws have been proposed in the literature since then. For example, the approach proposed by Coquel et al. [8] and used in 21] is based on two requirements: the enforcement of an entropy inequality and a linearly degenerate field associated with $\lambda=u_{I}$. The following closure laws comply with these two conditions:

$$
\begin{array}{rlrl}
u_{I} & =a u_{1}+(1-a) u_{2}, & a & =\frac{\chi \alpha_{1} \rho_{1}}{\chi \alpha_{1} \rho_{1}+(1-\chi) \alpha_{2} \rho_{2}} \\
p_{I}=b p_{1}+(1-b) p_{2}, & b & =\frac{(1-a) T_{2}}{a T_{1}+(1-a) T_{2}}
\end{array}
$$


with $\chi \in[0,1]$. We can notice that the case when $\chi=0$ (or $\chi=1$ ) corresponds to the closure laws of Baer and Nunziato [5] and the case when $\chi=\frac{1}{2}$ to the closure laws proposed in [8, 21. Those three choices $\left(\chi=0, \frac{1}{2}, 1\right)$ have been compared for steam-water transient flows in [36]. Other approaches can also be found in the literature [23, 31, 40, 7] and a wider class of models is introduced in appendix A of [36]. In the sequel, we use the closure laws proposed by Baer and Nunziato, detailed in equation (6).

System (1) may be recast in the following vectorial form:

$$
\partial_{t}(\mathbf{U})+\partial_{x}(\mathbf{F}(\mathbf{U}))+\mathbf{H}(\mathbf{U}) \partial_{x}\left(\alpha_{1}\right)=\mathbf{0}
$$

with

$$
\mathbf{U}=\left(\begin{array}{c}
\alpha_{1} \\
\alpha_{k} \rho_{k} \\
\alpha_{k} \rho_{k} u_{k} \\
\alpha_{k} \rho_{k} e_{k}
\end{array}\right), \quad \mathbf{F}(\mathbf{U})=\left(\begin{array}{c}
0 \\
\alpha_{k} \rho_{k} u_{k} \\
\alpha_{k} \rho_{k} u_{k}^{2}+\alpha_{k} p_{k} \\
\alpha_{k} \rho_{k} e_{k} u_{k}+\alpha_{k} p_{k} u_{k}
\end{array}\right) \text { and } \mathbf{H}(\mathbf{U})=\left(\begin{array}{c}
u_{I} \\
0 \\
(-1)^{k} p_{I} \\
(-1)^{k} p_{I} u_{I}
\end{array}\right)
$$

\subsection{Properties and Riemann Problem}

The homogeneous part of the Baer-Nunziato model has been first studied by Embid and Baer [19. We first recall its main mathematical properties and then tackle the Riemann problem:

Property 1 (Hyperbolicity and structure of waves). System (1) is hyperbolic, but not strictly hyperbolic. Indeed, it admits the following seven eigenvalues:

$$
\begin{aligned}
& \lambda_{1}=u_{1}-c_{1}, \quad \lambda_{2}=u_{1}, \quad \lambda_{3}=u_{1}+c_{1}, \\
& \lambda_{4}=u_{2}-c_{2}, \quad \lambda_{5,6}=u_{2}, \quad \lambda_{7}=u_{2}+c_{2}
\end{aligned}
$$

and associated righteigenvectors span the whole space $\mathbb{R}^{7}$ if $\left|u_{1}-u_{2}\right| \neq c_{1}$. Fields associated with eigenvalues $\lambda_{2,5,6}$ are linearly degenerate (LD) whereas fields associated with eigenvalues $\lambda_{1,4,3,7}$ are genuinely non linear (GNL).

Property 2 (Riemann invariants through LD waves).

- The Riemann invariants of the LD 2-wave are $\left\{\alpha_{1}, u_{1}, p_{1}, \rho_{2}, u_{2}, p_{2}\right\}$. Thus the volume fraction is constant across the wave and the 2-wave has the classical structure of a monophasic contact discontinuity with respect to phase 1.

- The 5 Riemann invariants of the LD 5,6-wave are the following:

$$
\begin{aligned}
& I_{1}=u_{2} \\
& I_{2}=\alpha_{1} \rho_{1}\left(u_{1}-u_{2}\right) \\
& I_{3}=\alpha_{1} p_{1}+\alpha_{2} p_{2}+\alpha_{1} \rho_{1}\left(u_{1}-u_{2}\right)^{2} \\
& I_{4}=\varepsilon_{1}+p_{1} / \rho_{1}+\left(u_{1}-u_{2}\right)^{2} / 2 \\
& I_{5}=s_{1}
\end{aligned}
$$

The 5,6-wave is the only one involving a jump of volume fraction therefore it will be referred as two-phase contact in the sequel. Although the structure of this wave is complex, some schemes [41, 42] directly use its 5 Riemann invariants when solving the Riemann problem. 
Property 3 (Jump conditions through GNL waves). Unique jump conditions hold within each isolated GNL field:

$$
\left\{\begin{array}{l}
{\left[\alpha_{k}\right]_{L}^{R}=0} \\
-\sigma\left[\rho_{k}\right]_{L}^{R}+\left[\rho_{k} u_{k}\right]_{L}^{R}=0 \\
-\sigma\left[\rho_{k} u_{k}\right]_{L}^{R}+\left[\rho_{k} u_{k}^{2}+p_{k}\right]_{L}^{R}=0 \\
-\sigma\left[\rho_{k} e_{k}\right]_{L}^{R}+\left[\rho_{k} e_{k} u_{k}+p_{k} u_{k}\right]_{L}^{R}=0
\end{array}\right.
$$

where $[\varphi]_{L}^{R}=\varphi_{R}-\varphi_{L}$ is the jump between the Left and Right states on each side of a shock wave traveling at speed $\sigma$.

The fact that the void fraction remains constant across the GNL waves is closely linked to the LD structure of the two-phase contact and leads to classical monophasic jump conditions through GNL waves.

Property 4 (Entropy of the system). Assume that $\left(u_{I}, p_{I}\right)$ comply with the following relation:

$$
\sum_{k} \frac{1}{T_{k}}(-1)^{k}\left(p_{k}-p_{I}\right)\left(u_{k}-u_{I}\right)=0
$$

Then smooth solutions of system (1) are such that:

$$
\partial_{t}\left(\sum_{k} \alpha_{k} \rho_{k} s_{k}\right)+\partial_{x}\left(\sum_{k} \alpha_{k} \rho_{k} s_{k} u_{k}\right)=0
$$

Proof. Smooth solutions of system (1) comply with:

$$
\partial_{t}\left(\alpha_{k} \rho_{k} s_{k}\right)+\partial_{x}\left(\alpha_{k} \rho_{k} s_{k} u_{k}\right)=\frac{1}{T_{k}}\left(p_{k}-p_{I}\right)\left(u_{k}-u_{I}\right) \partial_{x}\left(\alpha_{k}\right)
$$

So the equation of the total entropy $(12)$ is obtained thanks to the equation 111 by summing over the phases.

Thus the enforcement of the entropy inequality directly provides the closure law for the interfacial pressure $p_{I}$ thanks to equation (11) once the interfacial velocity $u_{I}$ has been set. If we set $u_{I}=u_{2}$ for example, we obtain $p_{I}=p_{1}$ which is the closure law proposed by Baer and Nunziato given in equation (6). Moreover, the same strategy has been used to provide closure laws with three, or more, phases [28, 38].

Thanks to all those theoretical properties, we address the Riemann problem for system (7):

$$
\left\{\begin{array}{l}
\partial_{t}(\mathbf{U})+\partial_{x}(\mathbf{F}(\mathbf{U}))+\mathbf{H}(\mathbf{U}) \partial_{x}\left(\alpha_{1}\right)=\mathbf{0} \\
\mathbf{U}(t=0, x)=\left\{\begin{array}{lll}
\mathbf{U}_{L}, & \text { if } & x<0 \\
\mathbf{U}_{R}, & \text { if } & x>0
\end{array}\right.
\end{array}\right.
$$

As pointed out in [4] (or in [21] in the case of other closure laws for interfacial quantities), the existence and uniqueness of the solution of such a problem is not guaranteed. Despite this, we can exhibit the general structure of a solution: it consists of several intermediate states connected by the 6 waves of the model. The previous properties provide the connection across each isolated wave, even shock waves thanks to the unique jump conditions across GNL waves detailed in property 3. An important difficulty of this Riemann problem lies in the fact that the wave-ordering is not known, different wave configurations are possible. Two cases are usually considered depending on the relative velocity $\left(u_{1}-u_{2}\right)$ : the case when $\left(u_{1}-u_{2}\right)^{2}<c_{1}^{2}$ holds is called the "subsonic" one and the opposite case, 

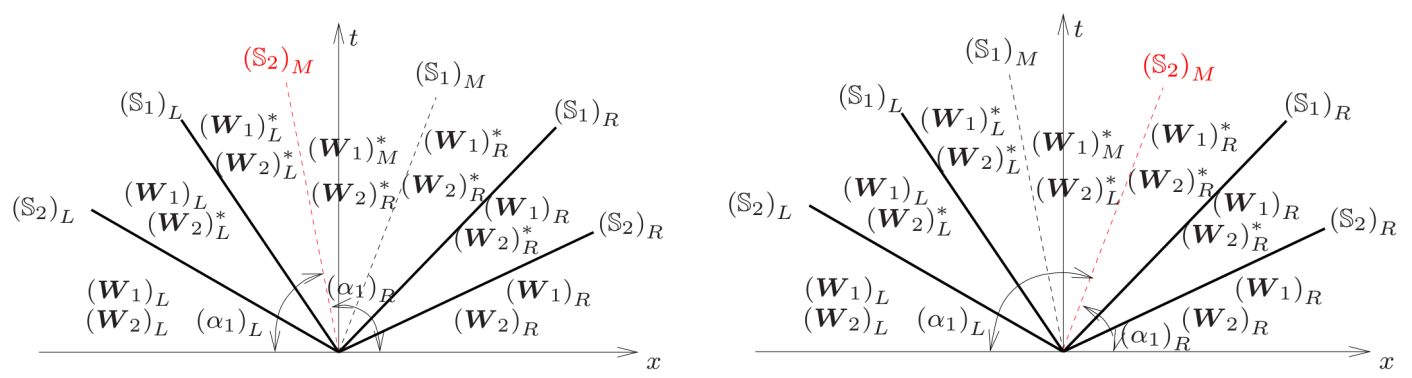

Figure 1: Structure of the exact solution of the Riemann problem for the Baer-Nunziato model in the "subsonic" situation. On the left, configuration (a) where $\left(\mathbb{S}_{2}\right)_{M} \leq\left(\mathbb{S}_{1}\right)_{M}$. On the right, configuration (b) where $\left(\mathbb{S}_{1}\right)_{M}<\left(\mathbb{S}_{2}\right)_{M}$.

when $\left(u_{1}-u_{2}\right)^{2}>c_{1}^{2}$, is called "supersonic". The subsonic case is considered to be more physicallyrelevant and we only consider the subsonic case in the sequel like many other authors in the literature [9, 42, 41]. Readers interested in the supersonic case are referred to [15, 16, 4].

In the subsonic case, two wave configurations, presented in figure 1, are possible. We denote by $\left(\mathbb{S}_{k}\right)_{L}$ (respectively $\left(\mathbb{S}_{k}\right)_{R}$ ) the GNL waves associated with $\lambda=u_{k}-c_{k}$ (respectively $\left.u_{k}+c_{k}\right)$. In the same way, we denote $\left(\mathbb{S}_{k}\right)_{M}$ the LD waves of the model. Thus, configuration (a) of figure 1 is the case $\left(\mathbb{S}_{2}\right)_{M} \leq\left(\mathbb{S}_{1}\right)_{M}$ and configuration (b) the opposite case $\left(\mathbb{S}_{1}\right)_{M}<\left(\mathbb{S}_{2}\right)_{M}$. For both configurations, the previous properties provide us the relations between the different intermediate states. Besides, as pointed out previously, the GNL waves have a similar structure as in the monophasic case therefore they don't interact with each other and we don't need to consider separate cases when $\left(\mathbb{S}_{1}\right)_{K}<\left(\mathbb{S}_{2}\right)_{K}$ or $\left(\mathbb{S}_{1}\right)_{K}>\left(\mathbb{S}_{2}\right)_{K}$ for $K=L, R$. The notation $\mathbf{W}_{k}$ used on figure 1 is defined by:

$$
\mathbf{W}_{k}=\left(\begin{array}{c}
\rho_{k} \\
u_{k} \\
p_{k}
\end{array}\right)
$$

Moreover, the volume fraction $\alpha_{k}$ only changes across the two-phase contact $\left(\mathbb{S}_{2}\right)_{M}$ as pointed out on the figure. We underline that the LD structure of this wave is crucial in order to obtain unique jump conditions. Indeed, this LD structure implies that the non-conservative products are not active in the other waves, especially the GNL waves (equation (10)). Thus, those non-conservative products are not "true" non-conservative products (i.e. active in GNL fields) which would have required particular definitions 13. As a consequence, shock solutions of the Riemann problems are defined in a nonambiguous way. This is of major importance from a computational point of view: without such properties, numerical grid converged shock solutions may differ due to the inner numerical diffusion of the scheme. To the authors' knowledge, this point has received very little attention in the literature on two-fluid models but striking numerical examples are available in [25] (pages 121-142).

\section{HLLC-type Riemann solver with approximated two-phase contact}

In this section, we introduce a HLLC-type Riemann solver to approximate the solutions of the BaerNunziato model based on the scheme proposed by Tokareva and Toro [42. The key idea of the present scheme is an approximation of the two-phase contact whereas it is treated using the thin layer approximation proposed by Schwendeman et al. [41] in the original HLLC-type solver [42. Thus, we first introduce the approximated two-phase contact then detail the intermediate states of the solution of the Riemann problem obtained thanks to the approximation and finally, we define the numerical flux of the solver based on the estimated states. 


\subsection{Approximated two-phase contact}

The Riemann invariants of the two-phase contact are recalled in equation (9). We introduce the coefficient $\widehat{\gamma}_{k}$ defined by the following:

$$
\widehat{\gamma}_{k}=\frac{\rho_{k} c_{k}^{2}}{p_{k}}
$$

In the particular case of the ideal gas equation of state (EOS), $\widehat{\gamma}_{k}$ is equal to the ratio of specific heats $\gamma_{k}$. Thanks to this notation, some Riemann invariants can be recast in the following form:

$$
\begin{aligned}
& I_{3}=\alpha_{1} \rho_{1}\left(\frac{1}{\widehat{\gamma}_{1}} c_{1}^{2}+\left(u_{1}-u_{2}\right)^{2}\right)+\frac{1}{\widehat{\gamma}_{2}} \alpha_{2} \rho_{2} c_{2}^{2} \\
& I_{4}=\varepsilon_{1}+\frac{1}{\widehat{\gamma}_{1}} c_{1}^{2}+\frac{1}{2}\left(u_{1}-u_{2}\right)^{2}
\end{aligned}
$$

In the subsonic case, we already assume that $\left(u_{1}-u_{2}\right)^{2}<c_{1}^{2}$. In order to approximate the two-phase contact, we propose a stronger hypothesis: $\left(u_{1}-u_{2}\right)^{2} \ll \frac{1}{\widehat{\gamma}_{1}} c_{1}^{2}$. This hypothesis is physically relevant since $u_{1}^{2} \ll c_{1}^{2}$ in a lot of practical configurations in the subsonic case. Moreover, the difference $\left(u_{1}-u_{2}\right)^{2}$ should be even smaller due to the velocity relaxation included in a complete model with convective terms and source terms such as drag effects [5]. Finally, $\frac{1}{\widehat{\gamma}_{1}} c_{1}^{2}$ can be considered to be in the same order of magnitude as $c_{1}^{2}$, particularly for most usual gases.

Thanks to the proposed hypothesis, the Riemann invariants of the wave are approximated in the following way:

$$
\begin{aligned}
& I_{1}=u_{2} \\
& I_{2}=\alpha_{1} \rho_{1}\left(u_{1}-u_{2}\right) \\
& I_{3} \approx \alpha_{1} p_{1}+\alpha_{2} p_{2} \\
& I_{4} \approx \varepsilon_{1}+p_{1} / \rho_{1} \\
& I_{5}=s_{1}
\end{aligned}
$$

Therefore, the specific internal enthalpy $\varepsilon_{1}+p_{1} / \rho_{1}$ of phase 1 seems to be a relevant approximation of $I_{4}$. However, the specific entropy $s_{1}\left(p_{1}, \rho_{1}\right)$ of phase 1 is the Riemann invariant $I_{5}$ of the wave. Preserving both is equivalent to the preservation of the primitive variables $\left(\rho_{1}, p_{1}\right)$. Thus, we define the approximated two-phase contact with the following new set of Riemann invariants:

$$
\begin{aligned}
& \widetilde{I_{1}}=u_{2} \\
& \widetilde{I_{2}}=\alpha_{1}\left(u_{1}-u_{2}\right) \\
& \widetilde{I_{3}}=\alpha_{1} p_{1}+\alpha_{2} p_{2} \\
& \widetilde{I_{4}}=p_{1} \\
& \widetilde{I_{5}}=\rho_{1}
\end{aligned}
$$

If we now focus on the consequences of this approximation in the structure of the solution of the 1D-Riemann problem (figure 1), we can notice that the approximation of the state $\left(\mathbf{W}_{1}\right)_{M}^{*}$ is directly obtained thanks to the states $\left(\mathbf{W}_{1}\right)_{K}^{*}, K=L, R$. For example, if we consider configuration (a), we have:

$$
\left(\mathbf{W}_{1}\right)_{M}^{*}=\left(\begin{array}{c}
\left(\rho_{1}\right)_{L}^{*} \\
\left(u_{1}\right)_{R}^{*} \\
\left(p_{1}\right)_{R}^{*}
\end{array}\right)
$$

whereas, in configuration (b), we get:

$$
\left(\mathbf{W}_{1}\right)_{M}^{*}=\left(\begin{array}{c}
\left(\rho_{1}\right)_{R}^{*} \\
\left(u_{1}\right)_{L}^{*} \\
\left(p_{1}\right)_{L}^{*}
\end{array}\right)
$$




\subsection{HLLC-type intermediate states}

This section is devoted to the estimations of the intermediate states of the solution of the Riemann problem (figure 1) using the approximation of the two-phase contact. In a HLLC-type approach, the intermediate states are obtained by using the averaged Rankine-Hugoniot relation across the GNL waves $\left(\mathbb{S}_{k}\right)_{K}(k=1,2$ and $K=L, R)$. The speeds of those waves need to be estimated a priori on the Left and Right states of the Riemann problem as in the HLL approach [27. Based on the work of Batten et al. [6] for the Euler equations, we use the following estimations, as in [15]:

$$
\begin{aligned}
& \left(\mathbb{S}_{k}\right)_{L}=\min \left(\left(u_{k}\right)_{L}-\left(c_{k}\right)_{L}, \hat{u_{k}}-\hat{c_{k}}\right) \\
& \left(\mathbb{S}_{k}\right)_{R}=\max \left(\left(u_{k}\right)_{R}+\left(c_{k}\right)_{R}, \hat{u_{k}}+\hat{c_{k}}\right)
\end{aligned} \quad, \quad k=1,2
$$

where $\hat{u_{k}}$ and $\hat{c_{k}}$ are the Roe-type average of the velocity and the speed of sound. We recall that the wave speeds of the LD waves $\left(\mathbb{S}_{k}\right)_{M}(k=1,2)$ do not need to be estimated a priori since they are directly given by the velocities of the intermediate states. If we consider the configuration (a) of figure 1. we have: $\left(\mathbb{S}_{1}\right)_{M}=\left(u_{1}\right)_{R}^{*}$ and $\left(\mathbb{S}_{2}\right)_{M}=\left(u_{2}\right)_{L}^{*}$.

The averaged Rankine-Hugoniot relations for the mass and momentum equations across the GNL waves provide the following HLLC-type intermediate states:

$$
\left\{\begin{array}{l}
\left(\rho_{k}\right)_{K}^{*}=\left(\rho_{k}\right)_{K} \frac{\left(\mathbb{S}_{k}\right)_{K}-\left(u_{k}\right)_{K}}{\left(\mathbb{S}_{k}\right)_{K}-\left(u_{k}\right)_{K}^{*}} \\
\left(p_{k}\right)_{K}^{*}=\left(p_{k}\right)_{K}+\left(\rho_{k}\right)_{K}\left(\left(\mathbb{S}_{k}\right)_{K}-\left(u_{k}\right)_{K}\right)\left(\left(u_{k}\right)_{K}^{*}-\left(u_{k}\right)_{K}\right)
\end{array}\right.
$$

for $k=1,2$ and $K=L, R$. They are similar to the intermediate states of the classical HLLC scheme 43] as expected since $\alpha_{1}$ is constant across the GNL waves. The intermediate states $\left(\mathbf{W}_{k}\right)_{K}^{*}$ can now be determined by using the preservation of the Riemann invariants of the approximated two-phase contact. The existence and uniqueness of those intermediate states are guaranteed by the following:

Proposition 1. Assume that the intermediate states of the Riemann problem comply with the preservation of the Riemann invariants of the approximated two-phase contact (16) and with the HLLC relations (18) where the wave speed estimates (17) are used. Then there exist unique intermediate states $\left(\mathbf{W}_{k}\right)_{K}^{*}(k=1,2$ and $K=L, M, R)$ of the Riemann problem for all initial conditions $\left(\mathbf{W}_{k}\right)_{K}(k=1,2$ and $K=L, R)$ and for all phasic equations of state (EOS).

Proof. Thanks to the HLLC relations $[18)$, we have $\left(u_{k}\right)_{K}^{*}$ as a linear function of $\left(p_{k}\right)_{K}^{*}$ :

$$
u_{k K}^{*}=u_{k K}+\frac{1}{\widetilde{c_{k K}}}\left(p_{k K}^{*}-p_{k K}\right) \quad \text { with } \quad \widetilde{c_{k K}}=\rho_{k K}\left(\mathbb{S}_{k K}-u_{k K}\right)
$$

where we use the short notation $\varphi_{k K}$ instead of $\left(\varphi_{k}\right)_{K}(k=1,2$ and $K=L, R)$ for simplicity. In order to determine the 4 intermediate pressures $p_{k K}^{*}$, we then need to use the Riemann invariants of the approximated two-phase contact from equation (16). Those Riemann invariants are also linear functions of the intermediate pressures and velocities therefore the intermediate pressures can be computed as the solutions of a linear system. We can notice that the pressure $p_{1}$ is a Riemann invariant $\widetilde{I}_{4}$ of the approximated contact so we introduce the notation $p_{1}^{*}=p_{1 L}^{*}=p_{1 R}^{*}$ and we end up with the following $3 \times 3$ linear system:

$$
\left(\begin{array}{ccc}
0 & \frac{1}{\widetilde{c_{2 L}}} & -\frac{1}{\widetilde{c_{2 R}}} \\
\frac{\alpha_{1 L}}{\widetilde{c_{1 L}}}-\frac{\alpha_{1 R}}{\widetilde{c_{1 R}}} & -\frac{\alpha_{1 L}}{\widetilde{c_{2 L}}} & \frac{\alpha_{1 R}}{\widetilde{c_{2 R}}} \\
\alpha_{1 L}-\alpha_{1 R} & \alpha_{2 L} & -\alpha_{2 R}
\end{array}\right)\left(\begin{array}{c}
p_{1}^{*} \\
p_{2 L}^{*} \\
p_{2 R}^{*}
\end{array}\right)=\left(\begin{array}{c}
R H S_{1} \\
R H S_{2} \\
0
\end{array}\right)
$$


with

$$
\begin{aligned}
& R H S_{1}=\frac{p_{2 L}}{\widetilde{c_{2 L}}}-u_{2 L}-\frac{p_{2 R}}{\widetilde{c_{2 R}}}+u_{2 R} \\
& R H S_{2}=\alpha_{1 L}\left(\frac{p_{1 L}}{\widetilde{c_{1 L}}}-u_{1 L}-\frac{p_{2 L}}{\widetilde{c_{2 L}}}+u_{2 L}\right)-\alpha_{1 R}\left(\frac{p_{1 R}}{\widetilde{c_{1 R}}}-u_{1 R}-\frac{p_{2 R}}{\widetilde{c_{2 R}}}+u_{2 R}\right)
\end{aligned}
$$

We underline the fact that this system is exactly the same for both wave configurations, (a) or (b) in figure 1. The determinant of the matrix can be easily computed:

$$
\Delta=\left(\frac{\alpha_{1 L}}{\widetilde{c_{1 L}}}-\frac{\alpha_{1 R}}{\widetilde{c_{1 R}}}\right)\left(\frac{\alpha_{2 R}}{\widetilde{c_{2 L}}}-\frac{\alpha_{2 L}}{\widetilde{c_{2 R}}}\right)-\frac{\left(\alpha_{1 L}-\alpha_{1 R}\right)^{2}}{\widetilde{c_{2 L}} \widetilde{c_{2 R}}}
$$

It is important to notice that $\widetilde{c_{k R}}>0$ and $\widetilde{c_{k L}}<0$ for both phases $(k=1,2)$ due to wave speed estimates given in equation (17). From that remark, we deduce that $\Delta>0$ so the system has a unique solution for all initial conditions $\left(\mathbf{W}_{k}\right)_{K}(k=1,2$ and $K=L, R)$. Once the intermediate pressures have been obtained, the intermediate states $\left(\mathbf{W}_{k}\right)_{K}^{*}$ can be fully determined thanks to equation (18). Then we also determine the wave configuration, (a) or (b) in figure 1 by looking at the sign of $\widetilde{I}_{2}$.

The existence and uniqueness of the intermediate states of the Riemann for all initial conditions is one of the major consequences of the approximation of the two-phase contact. It is important to recall that, without such an approximation, the connection through the two-phase contact is not possible for any Right and Left states as detailed in Appendix A. This approximation also has an important feature from the computational point of view since it leads to a linear system in order to determine the intermediate pressures whereas the original HLLC solver 42 needs to use an iterative procedure to solve a non-linear system. Finally, another major consequence of that approximation is that the analytical expression of the entropy $s_{1}$, which is an exact Riemann invariant of the wave $\left(I_{5}\right)$, is no longer required in the computation so the scheme can deal with any EOS.

\subsection{Numerical flux}

Thanks to the approximate solution of the Riemann problem proposed previously, we can introduce a Finite-Volume scheme based on this solution. This scheme is really close to the original HLLC scheme [42, the only difference lies in the intermediate states of the Riemann solver since the two-phase contact is approximated in our solver.

Let us assume a finite-volume mesh with volumes $\left[x_{i-\frac{1}{2}}, x_{i+\frac{1}{2}}\right] \times\left[t^{n}, t^{n+1}\right]$ of size $\Delta x \times \Delta t$. We define the cell average of the state vector $\mathbf{U}$ :

$$
\mathbf{U}_{i}^{n}=\frac{1}{\Delta x} \int_{x_{i-\frac{1}{2}}}^{x_{i+\frac{1}{2}}} \mathbf{U}\left(x, t^{n}\right) d x
$$

The integration of equation (7) gives us the following relation:

$$
\mathbf{U}_{i}^{n+1}=\mathbf{U}_{i}^{n}-\frac{\Delta t}{\Delta x}\left(\mathbf{F}_{i+\frac{1}{2}}^{n}-\mathbf{F}_{i-\frac{1}{2}}^{n}\right)-\frac{\Delta t}{\Delta x}\left(\mathbf{H}_{i+\frac{1}{2},-}^{n}-\mathbf{H}_{i-\frac{1}{2},+}^{n}\right)
$$

where we use the following discrete approximations of the fluxes:

$$
\mathbf{F}_{i+\frac{1}{2}}^{n} \approx \frac{1}{\Delta t} \int_{t^{n}}^{t^{n+1}} \mathbf{F}\left(\mathbf{U}\left(x_{i+\frac{1}{2}}, t\right)\right) d t
$$

and

$$
\left(\mathbf{H}_{i+\frac{1}{2},-}^{n}-\mathbf{H}_{i-\frac{1}{2},+}^{n}\right) \approx \frac{1}{\Delta t} \int_{t^{n}}^{t^{n+1}} \int_{x_{i-\frac{1}{2}}}^{x_{i+\frac{1}{2}}} \mathbf{H}(\mathbf{U}(x, t)) \partial_{x}\left(\alpha_{1}(x, t)\right) d x d t
$$


The computation of the fluxes is based on the approximate solution of a Riemann problem at each interface $x_{i+\frac{1}{2}}$ :

$$
\mathbf{F}_{i+\frac{1}{2}}^{n}=\widehat{\mathbf{F}}\left(\mathbf{U}_{i}^{n}, \mathbf{U}_{i+1}^{n}\right) \quad \text { and } \quad \mathbf{H}_{i+\frac{1}{2}, \pm}^{n}=\widehat{\mathbf{H}}_{ \pm}\left(\mathbf{U}_{i}^{n}, \mathbf{U}_{i+1}^{n}\right)
$$

Once the intermediate states $\left(\mathbf{W}_{1}\right)_{L}^{*},\left(\mathbf{W}_{1}\right)_{M}^{*},\left(\mathbf{W}_{1}\right)_{R}^{*},\left(\mathbf{W}_{2}\right)_{L}^{*},\left(\mathbf{W}_{2}\right)_{R}^{*}$ of the Riemann problem have been determined as explained previously, we use them to calculate the fluxes.

Let us first focus on the computation of the conservative part $\widehat{\mathbf{F}}\left(\mathbf{U}_{L}, \mathbf{U}_{R}\right)$. We use the following notation:

$$
\widehat{\mathbf{F}}\left(\mathbf{U}_{L}, \mathbf{U}_{R}\right)=\left(\begin{array}{c}
0 \\
\widehat{\mathbf{G}_{k}}\left(\mathbf{U}_{L}, \mathbf{U}_{R}\right)
\end{array}\right)
$$

The function $\widehat{\mathbf{G}_{2}}\left(\mathbf{U}_{L}, \mathbf{U}_{R}\right)$ is defined as follows [42]:

$$
\widehat{\mathbf{G}_{2}}\left(\mathbf{U}_{L}, \mathbf{U}_{R}\right)=\left\{\begin{array}{lll}
\left(\mathbf{G}_{2}\right)_{L} & \text { if } \quad 0 \leq\left(\mathbb{S}_{2}\right)_{L} \\
\left(\mathbf{G}_{2}\right)_{L}^{*}=\left(\mathbf{G}_{2}\right)_{L}+\left(\mathbb{S}_{2}\right)_{L}\left(\left(\mathbf{V}_{2}\right)_{L}^{*}-\left(\mathbf{V}_{2}\right)_{L}\right) & \text { if } \quad\left(\mathbb{S}_{2}\right)_{L} \leq 0 \leq\left(\mathbb{S}_{2}\right)_{M} \\
\left(\mathbf{G}_{2}\right)_{R}^{*}=\left(\mathbf{G}_{2}\right)_{R}+\left(\mathbb{S}_{2}\right)_{R}\left(\left(\mathbf{V}_{2}\right)_{R}^{*}-\left(\mathbf{V}_{2}\right)_{R}\right) & \text { if } \quad\left(\mathbb{S}_{2}\right)_{M} \leq 0 \leq\left(\mathbb{S}_{2}\right)_{R} \\
\left(\mathbf{G}_{2}\right)_{R} & \text { if } \quad\left(\mathbb{S}_{2}\right)_{R} \leq 0
\end{array}\right.
$$

In the case of phase $1, \widehat{\mathbf{G}_{1}}\left(\mathbf{U}_{L}, \mathbf{U}_{R}\right)$ depends on the wave configuration. For configuration (a) when $\left(\mathbb{S}_{2}\right)_{M} \leq\left(\mathbb{S}_{1}\right)_{M}$, we have:

$$
\widehat{\mathbf{G}_{1}}\left(\mathbf{U}_{L}, \mathbf{U}_{R}\right)= \begin{cases}\left(\mathbf{G}_{1}\right)_{L} & \text { if } 0 \leq\left(\mathbb{S}_{1}\right)_{L} \\ \left(\mathbf{G}_{1}\right)_{L}^{*}=\left(\mathbf{G}_{1}\right)_{L}+\left(\mathbb{S}_{1}\right)_{L}\left(\left(\mathbf{V}_{1}\right)_{L}^{*}-\left(\mathbf{V}_{1}\right)_{L}\right) & \text { if } \quad\left(\mathbb{S}_{1}\right)_{L} \leq 0 \leq\left(\mathbb{S}_{2}\right)_{M} \\ \left(\mathbf{G}_{1}\right)_{M}^{*}=\left(\mathbf{G}_{1}\right)_{R}^{*}+\left(\mathbb{S}_{1}\right)_{M}\left(\left(\mathbf{V}_{1}\right)_{M}^{*}-\left(\mathbf{V}_{1}\right)_{R}^{*}\right) & \text { if }\left(\mathbb{S}_{2}\right)_{M} \leq 0 \leq\left(\mathbb{S}_{1}\right)_{M} \\ \left(\mathbf{G}_{1}\right)_{R}^{*}=\left(\mathbf{G}_{1}\right)_{R}+\left(\mathbb{S}_{1}\right)_{R}\left(\left(\mathbf{V}_{1}\right)_{R}^{*}-\left(\mathbf{V}_{1}\right)_{R}\right) & \text { if }\left(\mathbb{S}_{1}\right)_{M} \leq 0 \leq\left(\mathbb{S}_{1}\right)_{R} \\ \left(\mathbf{G}_{1}\right)_{R} & \text { if }\left(\mathbb{S}_{1}\right)_{R} \leq 0\end{cases}
$$

and for configuration (b) when $\left(\mathbb{S}_{1}\right)_{M}<\left(\mathbb{S}_{2}\right)_{M}$ :

$$
\widehat{\mathbf{G}_{1}}\left(\mathbf{U}_{L}, \mathbf{U}_{R}\right)=\left\{\begin{array}{lll}
\left(\mathbf{G}_{1}\right)_{L} & \text { if } & 0 \leq\left(\mathbb{S}_{1}\right)_{L} \\
\left(\mathbf{G}_{1}\right)_{L}^{*}=\left(\mathbf{G}_{1}\right)_{L}+\left(\mathbb{S}_{1}\right)_{L}\left(\left(\mathbf{V}_{1}\right)_{L}^{*}-\left(\mathbf{V}_{1}\right)_{L}\right) & \text { if } & \left(\mathbb{S}_{1}\right)_{L} \leq 0 \leq\left(\mathbb{S}_{1}\right)_{M} \\
\left(\mathbf{G}_{1}\right)_{M}^{*}=\left(\mathbf{G}_{1}\right)_{L}^{*}+\left(\mathbb{S}_{1}\right)_{M}\left(\left(\mathbf{V}_{1}\right)_{M}^{*}-\left(\mathbf{V}_{1}\right)_{L}^{*}\right) & \text { if } & \left(\mathbb{S}_{1}\right)_{M} \leq 0<\left(\mathbb{S}_{2}\right)_{M} \\
\left(\mathbf{G}_{1}\right)_{R}^{*}=\left(\mathbf{G}_{1}\right)_{R}+\left(\mathbb{S}_{1}\right)_{R}\left(\left(\mathbf{V}_{1}\right)_{R}^{*}-\left(\mathbf{V}_{1}\right)_{R}\right) & \text { if } & \left(\mathbb{S}_{2}\right)_{M} \leq 0 \leq\left(\mathbb{S}_{1}\right)_{R} \\
\left(\mathbf{G}_{1}\right)_{R} & \text { if } & \left(\mathbb{S}_{1}\right)_{R} \leq 0
\end{array}\right.
$$

In equations (21)-23), we use the following notations (for $k=1,2$ and $K=L, R$ ):

$$
\begin{gathered}
\left(\mathbf{V}_{k}\right)_{K}=\left(\alpha_{k}\right)_{K}\left(\begin{array}{c}
\left(\rho_{k}\right)_{K} \\
\left(\rho_{k}\right)_{K}\left(u_{k}\right)_{K} \\
\left(\rho_{k}\right)_{K}\left(e_{k}\right)_{K}
\end{array}\right) \\
\left(\mathbf{G}_{k}\right)_{K}=\left(\alpha_{k}\right)_{K}\left(\begin{array}{c}
\left(\rho_{k}\right)_{K}\left(u_{k}\right)_{K} \\
\left(\rho_{k}\right)_{K}\left(\left(u_{k}\right)_{K}\right)^{2}+\left(p_{k}\right)_{K} \\
\left(\rho_{k}\right)_{K}\left(e_{k}\right)_{K}\left(u_{k}\right)_{K}+\left(p_{k}\right)_{K}\left(u_{k}\right)_{K}
\end{array}\right)
\end{gathered}
$$


Moreover the intermediate states are given by the HLLC relations:

$$
\left(\mathbf{V}_{k}\right)_{K}^{*}=\left(\alpha_{k}\right)_{K}\left(\rho_{k}\right)_{K}\left(\frac{\left(\mathbb{S}_{k}\right)_{K}-\left(u_{k}\right)_{K}}{\left(\mathbb{S}_{k}\right)_{K}-\left(\mathbb{S}_{k}\right)_{M}}\right)\left(\begin{array}{c}
1 \\
\left(\mathbb{S}_{k}\right)_{M} \\
\left(e_{k}\right)_{K}+\left(\left(\mathbb{S}_{k}\right)_{M}-\left(u_{k}\right)_{K}\right)\left(\left(\mathbb{S}_{k}\right)_{M}+\frac{\left(p_{k}\right)_{K}}{\left.\left(\rho_{k}\right)_{K}\left(\mathbb{S}_{k}\right)_{K}-\left(u_{k}\right)_{K}\right)}\right)
\end{array}\right)
$$

Finally, $\left(\mathbf{V}_{1}\right)_{M}^{*}$ is defined in the following way:

$$
\left(\mathbf{V}_{1}\right)_{M}^{*}=\left(\alpha_{1}\right)_{M}\left(\begin{array}{c}
\left(\rho_{1}\right)_{M}^{*} \\
\left(\rho_{1}\right)_{M}^{*}\left(u_{1}\right)_{M}^{*} \\
\left(\rho_{1}\right)_{M}^{*} \varepsilon_{1}\left(\left(\rho_{1}\right)_{M}^{*},\left(p_{1}\right)_{M}^{*}\right)+\frac{1}{2}\left(\rho_{1}\right)_{M}^{*}\left(\left(u_{1}\right)_{M}^{*}\right)^{2}
\end{array}\right)
$$

We recall that, in the case of configuration (a), we have:

$$
\left\{\begin{aligned}
\left(\alpha_{1}\right)_{M} & =\left(\alpha_{1}\right)_{R} \\
\left(\rho_{1}\right)_{M}^{*} & =\left(\rho_{1}\right)_{L}^{*} \\
\left(u_{1}\right)_{M}^{*} & =\left(u_{1}\right)_{R}^{*} \\
\left(p_{1}\right)_{M}^{*} & =\left(p_{1}\right)_{R}^{*}
\end{aligned}\right.
$$

and configuration (b) gives:

$$
\left\{\begin{aligned}
\left(\alpha_{1}\right)_{M} & =\left(\alpha_{1}\right)_{L} \\
\left(\rho_{1}\right)_{M}^{*} & =\left(\rho_{1}\right)_{R}^{*} \\
\left(u_{1}\right)_{M}^{*} & =\left(u_{1}\right)_{L}^{*} \\
\left(p_{1}\right)_{M}^{*} & =\left(p_{1}\right)_{L}^{*}
\end{aligned}\right.
$$

Then we focus on the computation of the non-conservative part $\widehat{\mathbf{H}}_{ \pm}\left(\mathbf{U}_{L}, \mathbf{U}_{R}\right)$. The main difficulty lies in the integration of the non-conservative product $p_{I} \partial_{x}\left(\alpha_{1}\right)$ across the two-phase contact as emphasized in [41. Thanks to our approximation of this wave given in equation (16), we have the following relation across the wave:

$$
p_{I} \partial_{x}\left(\alpha_{1}\right)=\partial_{x}\left(\alpha_{1} p_{1}\right)=-\partial_{x}\left(\alpha_{2} p_{2}\right)
$$

where we first use the preservation of $\widetilde{I}_{4}$ and then the preservation of $\widetilde{I}_{3}$. We underline the fact that the thin layer approximation proposed in [41] provides the same relation. Thus we treat those non-conservative products as in [41, 42, 15]:

$$
\widehat{\mathbf{H}}_{ \pm}\left(\mathbf{U}_{L}, \mathbf{U}_{R}\right)=\frac{1 \pm \operatorname{sign}\left[\left(\mathbb{S}_{2}\right)_{M}\right]}{2}\left(\begin{array}{c}
\left(\mathbb{S}_{2}\right)_{M}\left[\left(\alpha_{1}\right)_{R}-\left(\alpha_{1}\right)_{L}\right] \\
0 \\
(-1)^{k+1}\left[\left(\alpha_{2}\right)_{R}\left(p_{2}\right)_{R}^{*}-\left(\alpha_{2}\right)_{L}\left(p_{2}\right)_{L}^{*}\right] \\
(-1)^{k+1}\left(\mathbb{S}_{2}\right)_{M}\left[\left(\alpha_{2}\right)_{R}\left(p_{2}\right)_{R}^{*}-\left(\alpha_{2}\right)_{L}\left(p_{2}\right)_{L}^{*}\right]
\end{array}\right)
$$

Schwendeman et al. 41 also proposed a second-order extension of their Godunov solver. They proposed to use a classical minmod reconstruction of the primitive variables before applying the Riemann solver. Thus, the conservative part of the flux is obtained as well as the second-order contribution of the integral of the non-conservative terms near the two-phase contact. As detailed in [1], the second-order contribution of the integral of the non-conservative terms away from the two-phase contact remains and is also computed based on the solution obtained using the Riemann solver. Although it won't be considered in the sequel, a second-order extension of our scheme can be proposed using a similar approach based on our approximate Riemann solver. 


\section{Numerical results}

This section is dedicated to the assessment of the HLLC-type scheme introduced in the previous section. Due to the Approximation of the two-phase Contact, this solver will be referred as HLLAC in the sequel. We compare it with the Rusanov scheme [11] and the original HLLC-type solver from [42. on 1D as well as 2D test-cases. Those three schemes have been implemented in the fast transient dynamics software Europlexus [1, 15] which has been used for all the following computations.

General equations of states have been used so far but we need to specify them for the numerical test-cases. We consider that both phases are governed by the Stiffened Gas EOS as in most numerical tests from the literature:

$$
\varepsilon_{k}\left(\rho_{k}, p_{k}\right)=\frac{p_{k}+\gamma_{k} p_{k}^{\infty}}{\left(\gamma_{k}-1\right) \rho_{k}}
$$

Thus, two thermodynamic constants $\gamma_{k}$ and $p_{k}^{\infty}$ need to be defined for both phases in each test-case.

\subsection{D test-cases}

Seven 1D Riemann problems have been chosen in order to assess the accuracy, the robustness and the efficiency of the present scheme. Most of them have been proposed in 14] where analytical solutions are provided with a maximum accuracy allowing to carry out grid convergence studies. The SG EOS parameters for all test-cases (except test 7) are gathered in table 1 . The initial conditions consist of two constant states, detailed in table 2, separated by a discontinuity at $x_{0}=0.5$ (except for test 4 ). The last 1D test-case has been chosen to assess the ability of the proposed solver to deal with complex EOS. In each test, we consider the domain $[0,1]$ with transmissive boundary conditions at both ends. All computations have been carried out with a Courant number $C_{C F L}=\max \left(\lambda_{k}\right) \frac{\Delta t}{\Delta x}=0.5$.

Table 1: SG EOS parameters for the 1D Riemann problems

\begin{tabular}{ccccc}
\hline & $\boldsymbol{\gamma}_{\mathbf{1}}$ & $\boldsymbol{\gamma}_{\mathbf{2}}$ & $\boldsymbol{p}_{\mathbf{1}}^{\boldsymbol{\infty}}$ & $\boldsymbol{p}_{\mathbf{2}}^{\boldsymbol{\infty}}$ \\
\hline Test $\mathbf{1}$ & 3 & 2 & 0. & 0. \\
\hline Test 2 & 3 & 2 & 0. & 0. \\
\hline Test $\mathbf{3}$ & 1.4 & 3 & 0. & 10. \\
\hline Test $\mathbf{4}$ & 1.4 & 3 & 0. & 100. \\
\hline Test 5 & 3 & 2 & 0. & 0. \\
\hline Test $\mathbf{6}$ & 1.4 & 3 & 0. & 0. \\
\hline
\end{tabular}

Table 2: Initial conditions for the 1D Riemann problems

\begin{tabular}{|c|c|c|c|c|c|c|c|c|}
\hline & & $\alpha_{2}$ & $\rho_{1}$ & $u_{1}$ & $p_{1}$ & $\rho_{2}$ & $u_{2}$ & $p_{2}$ \\
\hline \multirow{2}{*}{ Test 1 [14] } & Left state & 0.8 & 1.25 & 0.1837722 & 1. & 1.5185185 & 0.3110988 & 1.2 \\
\hline & Right state & 0.2 & 1.2539534 & 0.2793675 & 1.0095183 & 0.5 & 0.3110988 & 1.7771419 \\
\hline \multirow{2}{*}{ Test 2 [14] } & Left state & 0.8 & 1. & 0.5 & 0.5 & 1. & 0.8 & 0.5 \\
\hline & Right state & 0.2 & 1.2539534 & 0.2793675 & 1.0095183 & 0.5 & 0.3110988 & 1.7771419 \\
\hline \multirow{2}{*}{ Test 3 [14] } & Left state & 0.6 & 1.4 & 0 . & 1. & 1.4 & 0 . & 2. \\
\hline & Right state & 0.3 & 1. & 0 . & 1. & 1. & 0 . & 3. \\
\hline \multirow{2}{*}{ Test 4 42] } & Left state & 0.7 & 1. & -19.5975 & 1000. & 1. & -19.5975 & 1000. \\
\hline & Right state & 0.2 & 1. & -19.5975 & 0.01 & 1. & -19.5975 & 0.01 \\
\hline \multirow{2}{*}{ Test 5} & Left state & $1-10^{-6}$ & 1.25 & 0.1837722 & 1. & 1.5185185 & 0.3110988 & 1.2 \\
\hline & Right state & $10^{-6}$ & 1.25421479 & 0.311098673 & 1.01014963 & 0.5 & 0.3110988 & 189851.199 \\
\hline \multirow{2}{*}{ Test 6 41] } & Left state & 0.5 & 1. & 0. & 2. & 2. & 0 . & 5. \\
\hline & Right state & $10^{-6}$ & 1.8 & 0 . & 4. & 1.7829 & 0.2972 & 3.5422 \\
\hline
\end{tabular}




\subsubsection{Test 1: Isolated two-phase contact}

This test proposed in 14] consists of an isolated two-phase contact discontinuity. To build such a testcase, a Left state needs to be set first allowing the evaluation of the exact Riemann invariants of the wave, given in equation (9). Then the preservation of those Riemann invariants provides us non-linear equations that need to be solved in order to obtain the Right state (see [11 for more details). The exact solution of the corresponding Riemann problem is an isolated two-phase contact discontinuity since the Riemann invariants of the wave are exactly preserved. Thus, this test-case appears to be relevant to assess the consequence of the key approximation of the HLLAC scheme. Figure 2 shows the comparison between the analytical solution and the numerical solutions obtained with the three different schemes on 1000 cells. As expected, the Rusanov scheme is very diffusive (see the results on $\alpha_{2}$ for example) compared to the two others which seem to give a similar diffusion at the interface. We can also notice some "ghost waves" with inaccurate intermediate states on the numerical results of the HLLAC scheme linked to the approximation of the two-phase contact (particularly on $\rho_{1}$ and $p_{1}$ ). Indeed, the Riemann invariants of the approximated two-phase contact (equation (16p) are not preserved between the Left and Right states, unlike the exact ones, therefore additional waves appear. In order to check if those "ghost waves" disappear when refining the grid, a convergence study has been carried out and results are provided in figure 3. For all schemes, we observed the expected rate of convergence $1 / 2$ for first-order schemes due to the occurrence of a contact discontinuity. Moreover, the HLLC scheme appears to be the most accurate solver for all variables as expected, whereas the Rusanov scheme is the least accurate one. We notice that the accuracy of the HLLAC scheme is really close to the accuracy of the HLLC scheme for $\left(\alpha_{2}, \rho_{2}, p_{2}, u_{1}\right)$. For the other variables, $\left(\rho_{1}, p_{1}, u_{2}\right)$, the accuracy of the HLLAC scheme is closer to the one of the Rusanov scheme which is not surprising since those three variables correspond to some Riemann invariants of the approximated two-phase contact.

\subsubsection{Test 2: Two-phase contact with shock waves}

The second test is built on the previous one in [14]: one shock wave propagating to the left has been added to the two-phase contact in each phase. The Right state is the same as in the previous test. The new Left state is obtained thanks to jump conditions 10 with the Left state from the previous test-case. Thus, a classical monophasic (i.e. complying with the jump conditions of the Euler equations) shock wave propagating to the left appears in each phase in addition to the two-phase contact. Figure 4 provides the comparison between the analytical solution and the numerical ones. Convergence studies have also been carried out on this test and are presented in figure 5 . The first remark is that the results of HLLAC and HLLC schemes are very close showing that the proposed approximation of the two-phase contact has a minor impact on the overall accuracy of the solver on this test-case. Differences between those two schemes can only be seen on very fine grids. Compared to those schemes, the Rusanov one appears to be very diffusive and less accurate. Except for $u_{2}$, the expected order of convergence $1 / 2$ is retrieved. In the case of $u_{2}$, the observed order of convergence is 1 due to the fact that it is constant across the contact discontinuity and therefore exhibits only a shock wave.

An efficiency study is also carried out on this test to assess the computational cost of the proposed scheme. Thus figure 6 shows the error on $\alpha_{2}$ from the grid convergence study versus the CPU time of the corresponding computation for the three different schemes. At a given error, we can see that the Rusanov scheme requires a higher computational cost than the other schemes although it is the least expensive scheme on a given mesh. Among both other schemes, the HLLAC one has a lower computational cost than the HLLC one due to the fact that it does not require the use of an iterative method for each local Riemann problem. More precisely, table 3 provides the computational cost of each scheme for a given error $\left(\operatorname{err}\left(\alpha_{2}\right)=10^{-4}\right)$. The Rusanov scheme appears to be 40 times more expensive than the HLLAC scheme whereas the HLLC one is 2.5 times more expensive than the HLLAC scheme. This latter observation is linked to the fact that 2 iterations are used in the Newton's method involved in the HLLC scheme as suggested in [42]. 

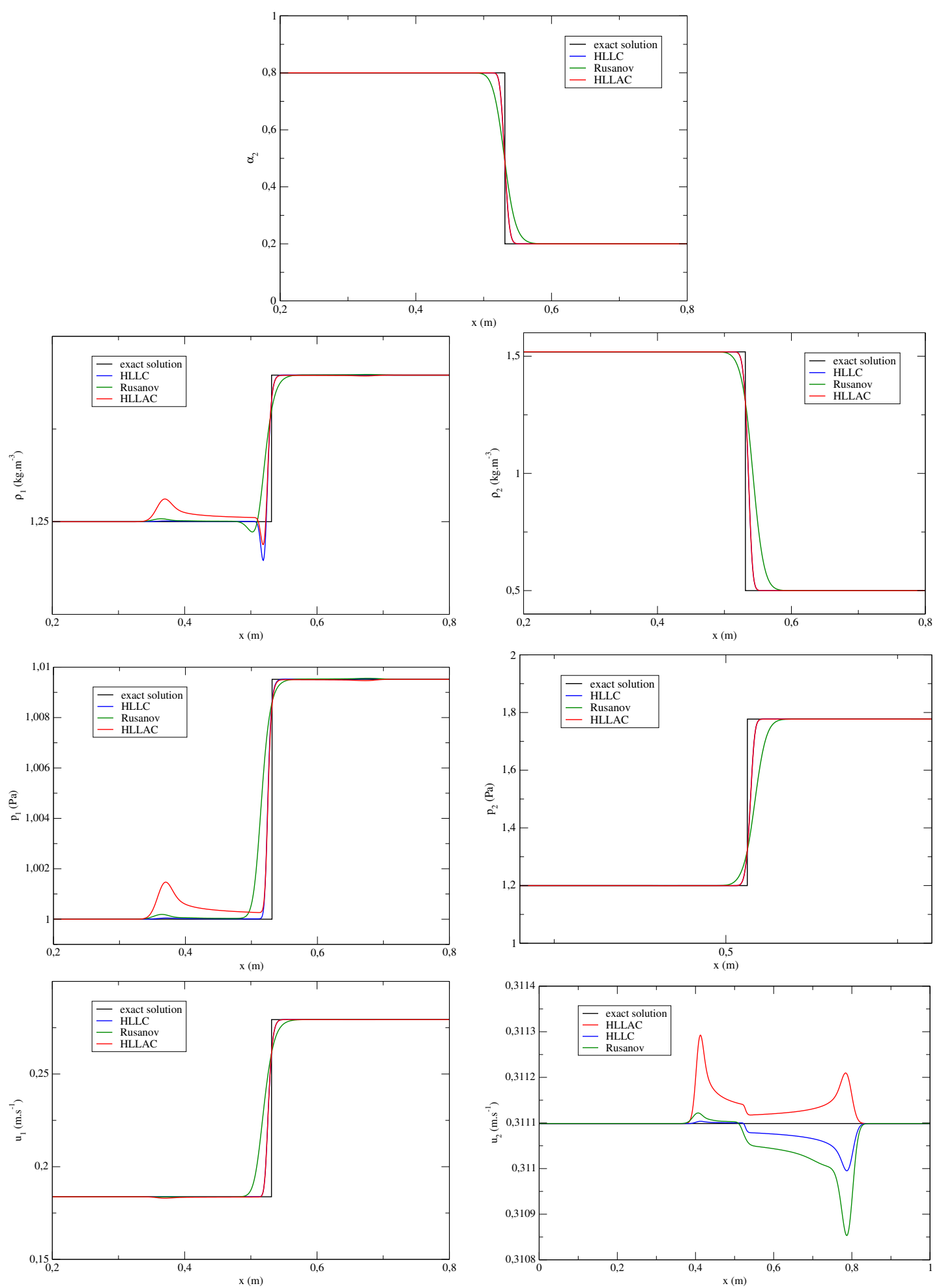

Figure 2: Comparison between analytical and numerical solutions on Test 1 with 1000 cells at time $t_{f}=0.1 \mathrm{~s}$. From the top to the bottom right: $\alpha_{2}, \rho_{1}, \rho_{2}, p_{1}, p_{2}, u_{1}, u_{2}$ vs $x$. 

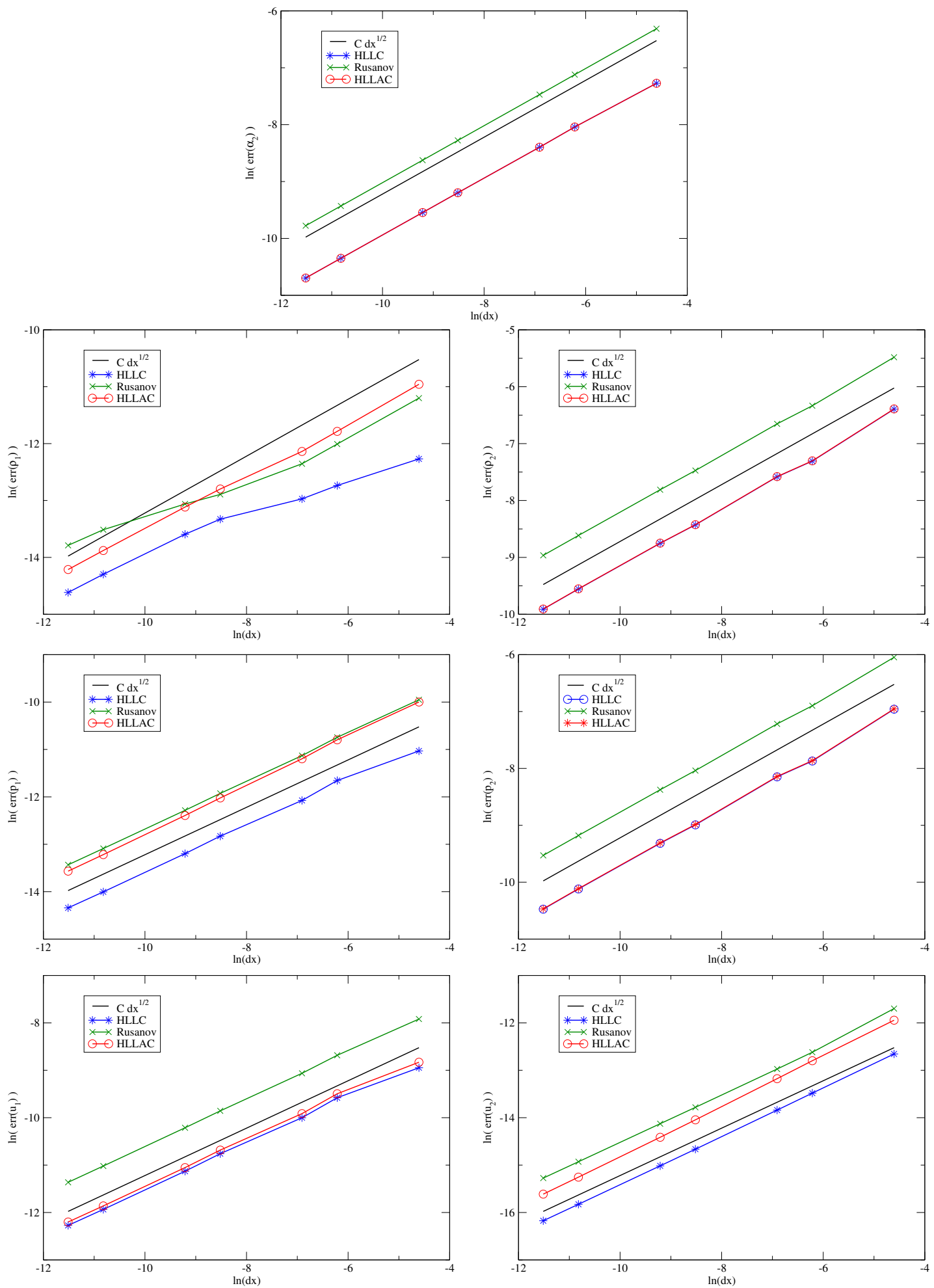

Figure 3: Convergence study on Test 1 at time $t_{f}=0.1 \mathrm{~s}$. From the top to the bottom right: L1 norm of the error on $\alpha_{2}, \rho_{1}, \rho_{2}, p_{1}, p_{2}, u_{1}, u_{2}$ vs $\Delta x$. 

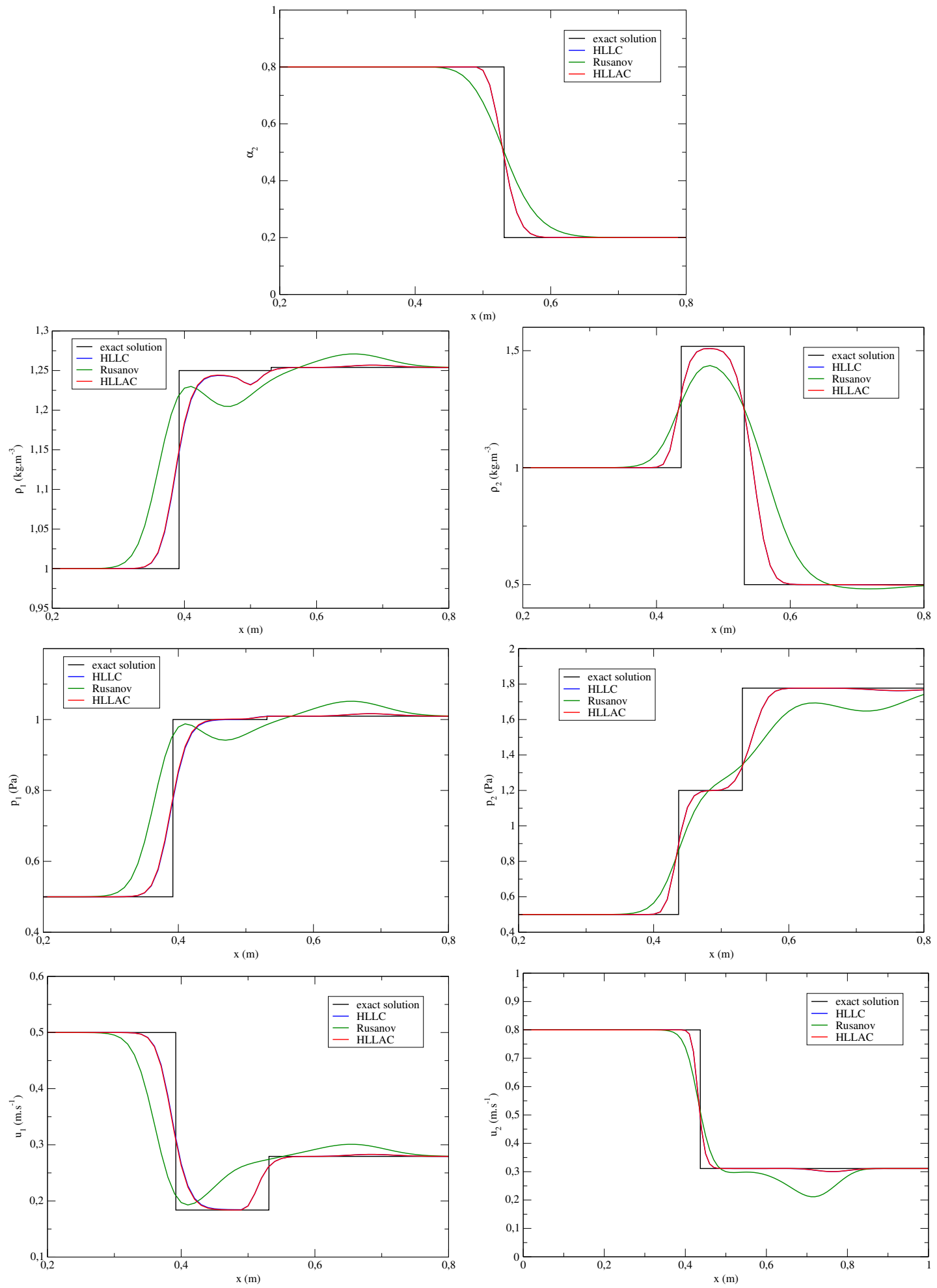

Figure 4: Comparison between analytical and numerical solutions on Test 2 with 100 cells at time $t_{f}=0.1 \mathrm{~s}$. From the top to the bottom right: $\alpha_{2}, \rho_{1}, \rho_{2}, p_{1}, p_{2}, u_{1}, u_{2}$ vs $x$. 

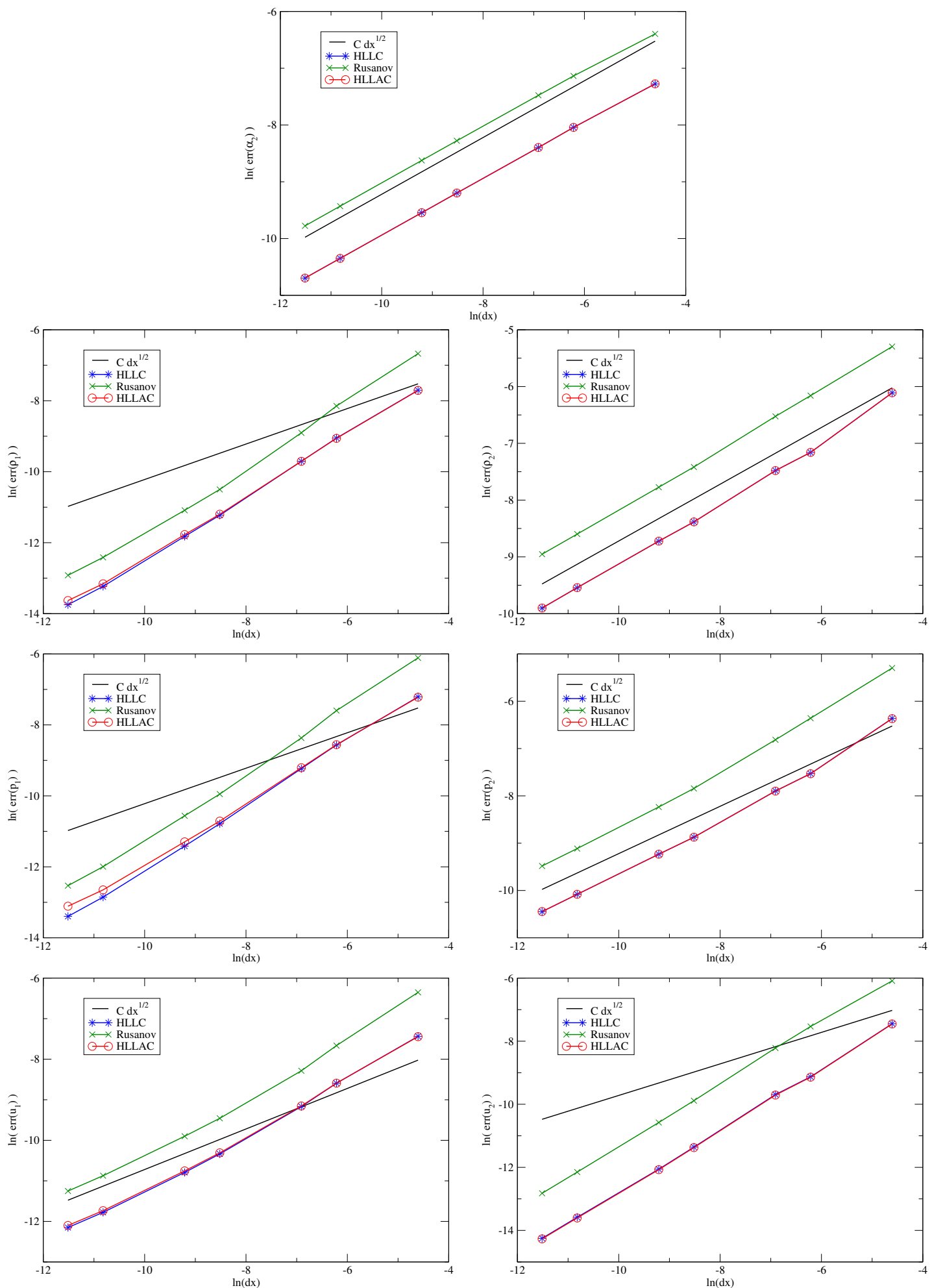

Figure 5: Convergence study on Test 2 at time $t_{f}=0.1 \mathrm{~s}$. From the top to the bottom right: L1 norm of the error on $\alpha_{2}, \rho_{1}, \rho_{2}, p_{1}, p_{2}, u_{1}, u_{2}$ vs $\Delta x$. 


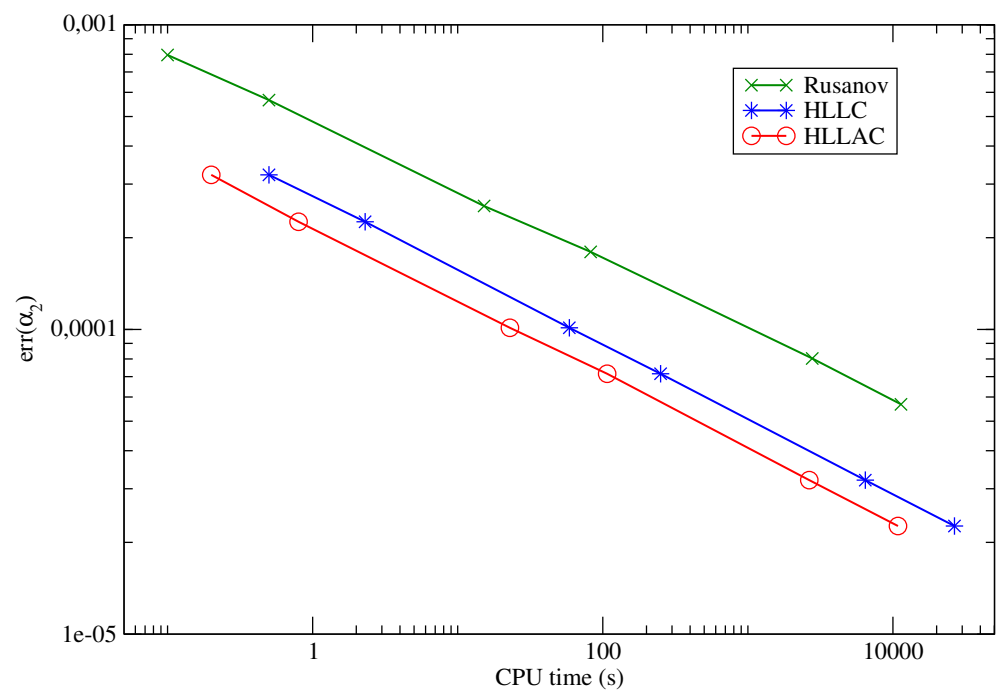

Figure 6: Efficiency study on Test 2 at time $t_{f}=0.1 s:$ L1 norm of the error on $\alpha_{2}$ vs CPU time of the computation.

Table 3: Computational cost of the three schemes for $\operatorname{err}\left(\alpha_{2}\right)=10^{-4}$.

\begin{tabular}{lccc}
\hline Scheme & HLLC & Rusanov & HLLAC \\
\hline CPU time (s) & 61 & 1055 & 24 \\
\hline
\end{tabular}

\subsubsection{Test 3: Stationary two-phase contact}

The third test-case consists of a stationary isolated two-phase contact proposed in [14. The key point of this test is to assess that the HLLAC scheme keeps the property of the HLLC scheme to exactly preserve this wave. Whereas the two-phase contact is diffused by the Rusanov scheme, figure 7 shows that the HLLAC does preserve it as expected since the neglected terms of the Riemann invariants of this wave are equal to zero in this particular test-case.

\subsubsection{Test 4: A general Riemann problem}

This test has been used in [14] but was initially proposed in [42. The exact solution of the Riemann problem is composed by the 6 waves of the model including strong shock waves. Unlike the other tests, the initial discontinuity is located at $x_{0}=0.8$. The comparison of the analytical and numerical solutions is shown in figure 8 . Once again, the results of the HLLAC and HLLC scheme are very close. This test-case is particularly severe and we can observe that the accuracy of the Rusanov scheme is not sufficient to retrieve the intermediate states on coarse grids.

\subsubsection{Test 5: Isolated two-phase contact with vanishing phases}

Test 5 has been designed in the same way as test 1 so that it consists of an isolated two-phase contact. However it is also a test with vanishing phases in both states ( $\alpha_{k}$ close to 0 or 1 ) in order to assess the robustness of the schemes. Figure 9 provides the analytical solution and the numerical solutions obtained with the Rusanov and the HLLAC schemes. Indeed the HLLC scheme was not robust enough for this test since it did not preserve the positivity of pressures. We observe that the accuracy of the Rusanov scheme is very poor on this severe test, especially on phase 2 . We also study the convergence of the two schemes on this test-case. Surprising results for the HLLAC scheme on coarse grids (for 

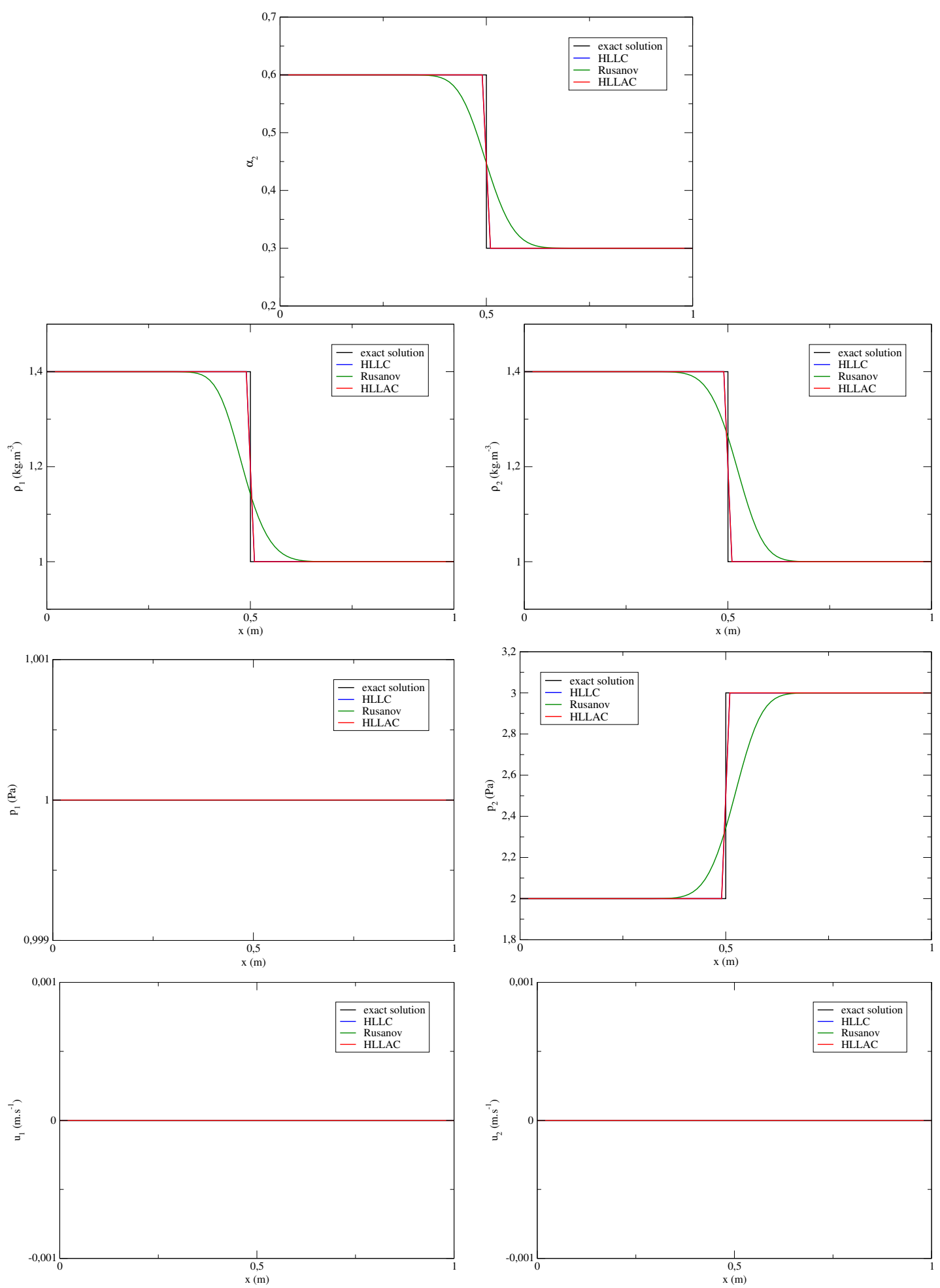

Figure 7: Comparison between analytical and numerical solutions on Test 3 with 100 cells at time $t_{f}=0.05 \mathrm{~s}$. From the top to the bottom right: $\alpha_{2}, \rho_{1}, \rho_{2}, p_{1}, p_{2}, u_{1}, u_{2}$ vs $x$. 

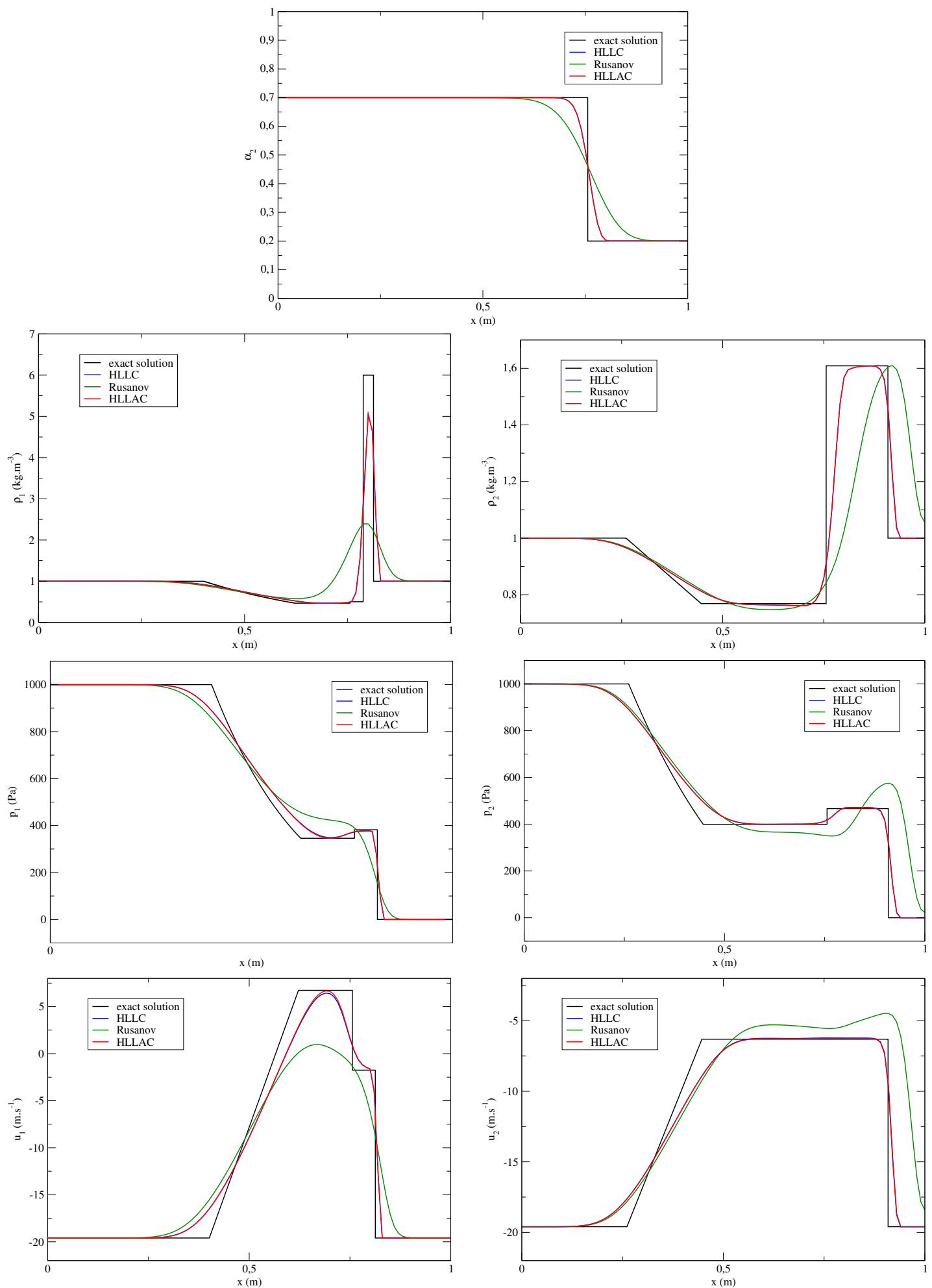

Figure 8: Comparison between analytical and numerical solutions on Test 4 with 100 cells at time $t_{f}=0.007 \mathrm{~s}$. From the top to the bottom right: $\alpha_{2}, \rho_{1}, \rho_{2}, p_{1}, p_{2}, u_{1}, u_{2}$ vs $x$. 
phase 1) are observed on figure 10. Nevertheless we retrieve the order of convergence of $1 / 2$ for both schemes except in the case of the error on $u_{2}$ which remains stable, close to round-off errors.

\subsubsection{Test 6: A general Riemann problem with vanishing phases}

Test 6 is a general Riemann problem with vanishing phases proposed in [41]. The exact solution of the problem is composed by five of the six waves of the model, only the GNL wave $\left(\mathbb{S}_{2}\right)_{R}$ is not present. Figure 11 provides the comparison between the analytical solution and the numerical solutions of the three schemes. We still observe similar results with the HLLAC and HLLC schemes, even on "ghost waves" in phase 2. Moreover the diffusion of the Rusanov scheme, which is striking on $\alpha_{2}$, leads to a poor accuracy when compared to the two other schemes.

\subsubsection{Test 7: Complex Equations Of State}

This last Riemann problem has been chosen to assess the ability of the proposed solver to deal with any Equation Of State. Thus, a test-case from [40, which studies the interaction between gaseous detonation products (phase 1) and a copper plate (phase 2), is considered. The detonation products are governed by the Jones-Wilkins-Lee (JWL) EOS while the copper is governed by the Cochran-Chan (CC) EOS. Both EOS can be written in the Mie-Gruneisen form:

$$
\varepsilon_{k}\left(\rho_{k}, p_{k}\right)=\frac{p_{k}-\Pi_{k}\left(\rho_{k}\right)}{\Gamma_{k} \rho_{k}}+Q_{k}\left(\rho_{k}\right)
$$

where the functions $\Pi_{k}\left(\rho_{k}\right)$ and $Q_{k}\left(\rho_{k}\right)$ need to be specify. In the case of the JWL EOS, they depend on the Chapman-Jouguet state (CJ):

$$
\begin{aligned}
\Pi_{1}(\rho)= & A \exp \left(-R_{1} \frac{\rho_{1}^{r e f}}{\rho}\right)+B \exp \left(-R_{2} \frac{\rho_{1}^{r e f}}{\rho}\right) \\
Q_{1}(\rho)= & \frac{A}{\rho_{1}^{r e f} R_{1}} \exp \left(-R_{1} \frac{\rho_{1}^{r e f}}{\rho}\right)+\frac{B}{\rho_{1}^{r e f} R_{2}} \exp \left(-R_{2} \frac{\rho_{1}^{r e f}}{\rho}\right) \\
& -\frac{A}{\rho_{1}^{r e f} R_{1}} \exp \left(-R_{1} \frac{\rho_{1}^{r e f}}{\rho_{C J}}\right)-\frac{B}{\rho_{1}^{r e f} R_{2}} \exp \left(-R_{2} \frac{\rho_{1}^{r e f}}{\rho_{C J}}\right)-\frac{p_{C J}-\Pi_{1}\left(\rho_{C J}\right)}{\Gamma_{1} \rho_{C J}}+e_{C J}
\end{aligned}
$$

In the case of the CC EOS, we have:

$$
\begin{aligned}
& \Pi_{2}(\rho)=A_{1}\left(\frac{\rho}{\rho_{2}^{r e f}}\right)^{E_{1}}-A_{2}\left(\frac{\rho}{\rho_{2}^{r e f}}\right)^{E_{2}} \\
& Q_{2}(\rho)=\frac{A_{1}}{\rho_{2}^{r e f}\left(E_{1}-1\right)}\left(\left(\frac{\rho}{\rho_{2}^{r e f}}\right)^{E_{1}-1}-1\right)-\frac{A_{2}}{\rho_{2}^{r e f}\left(E_{2}-1\right)}\left(\left(\frac{\rho}{\rho_{2}^{r e f}}\right)^{E_{2}-1}-1\right)-e_{0}
\end{aligned}
$$

Many thermodynamical parameters are required for both EOS and they are provided in table 4 Initially, the detonation products are at the Chapman-Jouguet state and the copper is under atmospheric conditions, as recalled in table 5. Only the HLLAC scheme is considered in this test and different meshes are used. Figure 12 provides the numerical results on the fraction $\alpha_{2}$ and the mean variables $\rho=\alpha_{1} \rho_{1}+\alpha_{2} \rho_{2}, u=\frac{1}{\rho}\left(\alpha_{1} \rho_{1} u_{1}+\alpha_{2} \rho_{2} u_{2}\right), p=\alpha_{1} p_{1}+\alpha_{2} p_{2}$ at time $t_{f}=73 \times 10^{-6} s$. The solution of this test-case consists of a rarefaction wave propagating to the left in the detonation gases, a two-phase contact propagating to the right and a shock wave propagating to the right in the copper. Due to the complexity of the considered EOS, no exact solution is available for this test but the comparison of the numerical solutions on the different meshes shows that the different waves and intermediate states are accurately captured by the HLLAC scheme when refining the grid. This numerical solution is in 

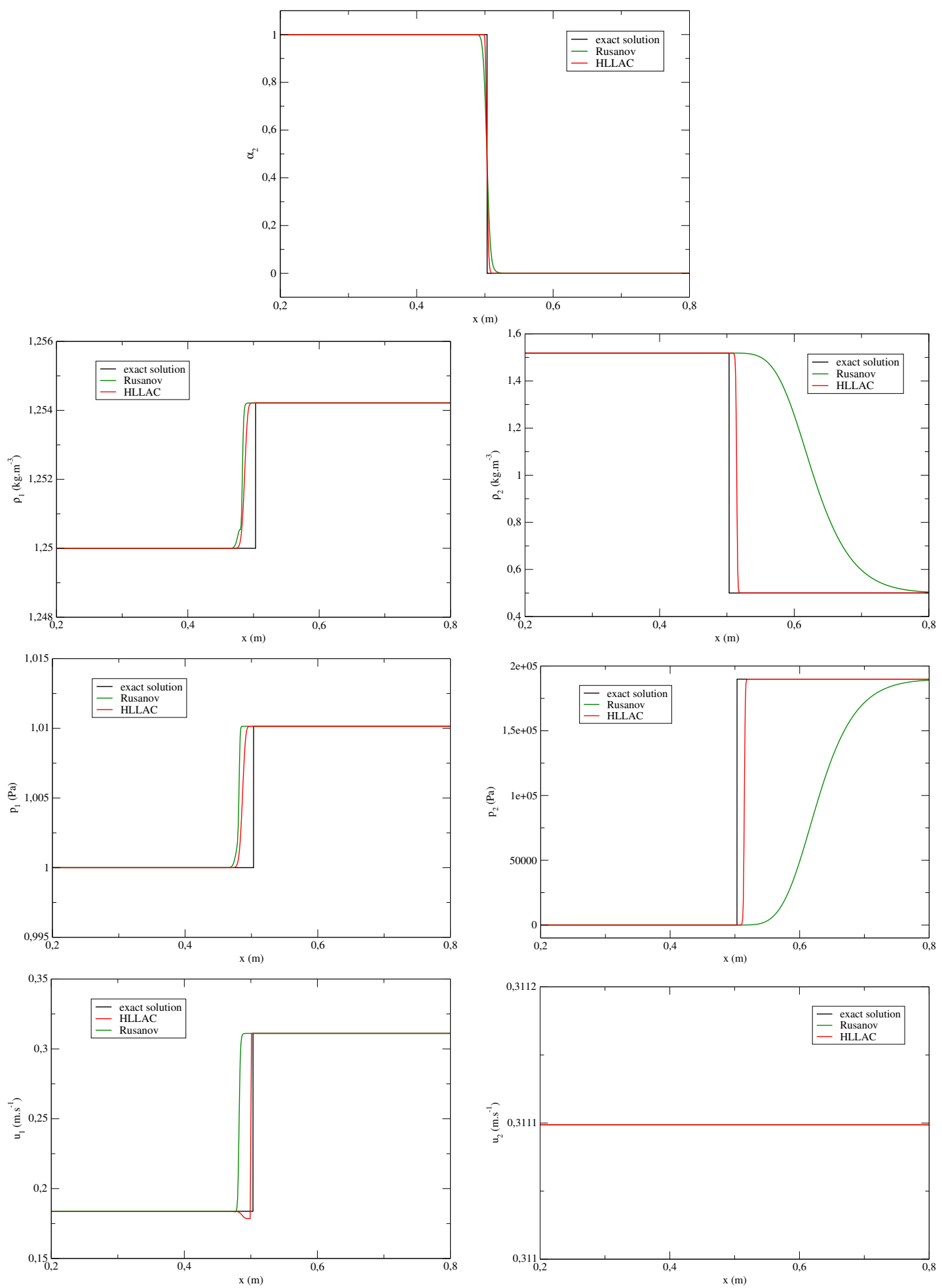

Figure 9: Comparison between analytical and numerical solutions on Test 5 with 1000 cells at time $t_{f}=0.01 \mathrm{~s}$. From the top to the bottom right: $\alpha_{2}, \rho_{1}, \rho_{2}, p_{1}, p_{2}, u_{1}, u_{2}$ vs $x$. 

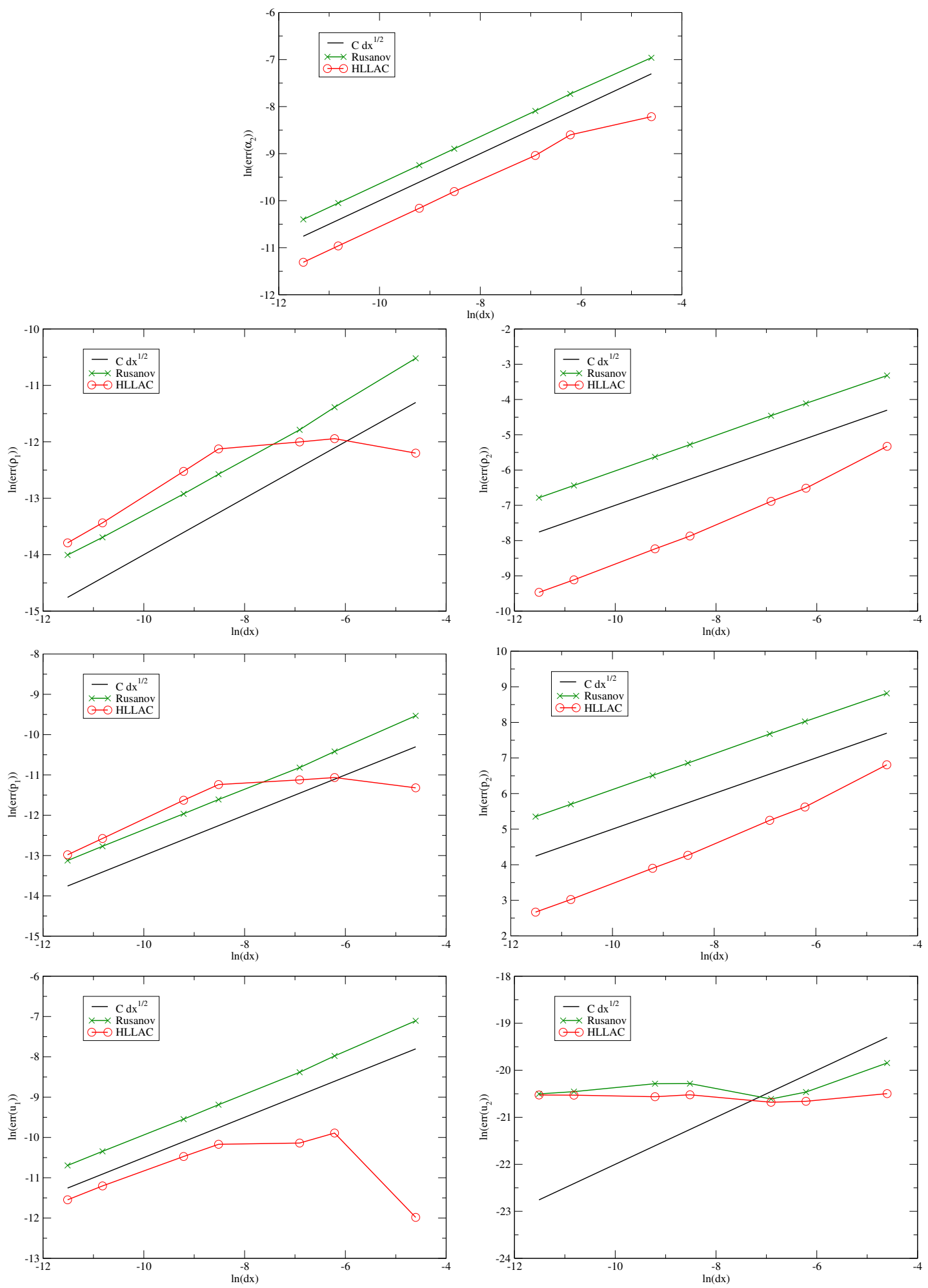

Figure 10: Convergence study on Test 5 at time $t_{f}=0.01 \mathrm{~s}$. From the top to the bottom right: L1 norm of the error on $\alpha_{2}, \rho_{1}, \rho_{2}, p_{1}, p_{2}, u_{1}, u_{2}$ vs $\Delta x$. 

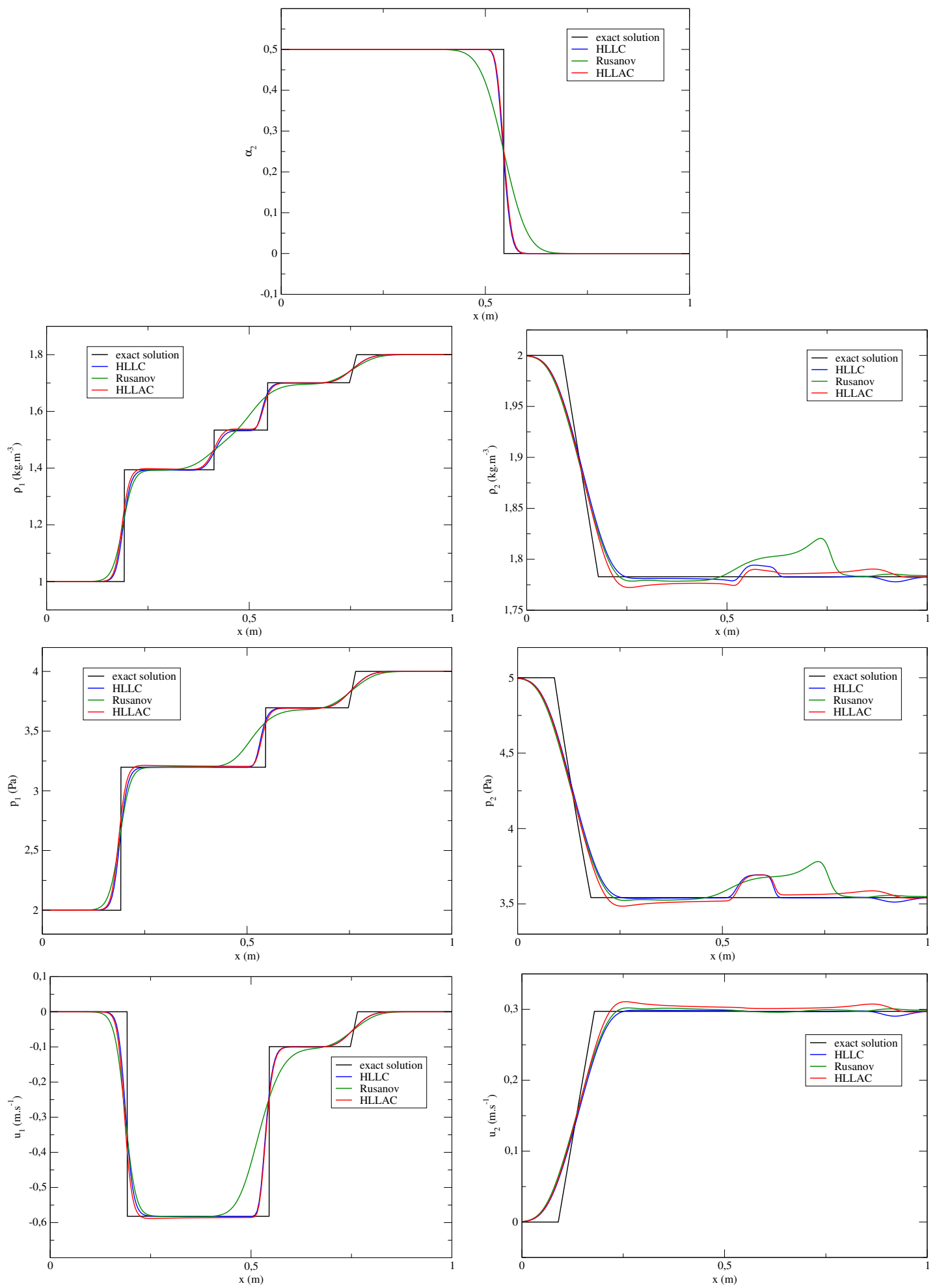

Figure 11: Comparison between analytical and numerical solutions on Test 6 with 200 cells at time $t_{f}=0.15 \mathrm{~s}$. From the top to the bottom right: $\alpha_{2}, \rho_{1}, \rho_{2}, p_{1}, p_{2}, u_{1}, u_{2}$ vs $x$. 
agreement with the one provided in [40] (Figure 11 page 454). Since we use a first-order scheme, more diffusion is observed in our numerical results. We also recall that Saurel \& Abgrall [40] do not consider the Baer-Nunziato closure laws (equation (6) ) and use instantaneous pressure and velocity relaxations. This test-case demonstrates the ability of the HLLAC to deal with complex EOS, even in the presence of vanishing phases and violent pressure waves.

Table 4: JWL and CC EOS parameters for Test 7

\begin{tabular}{lc|lc}
\hline \multicolumn{2}{c|}{ JWL EOS } & \multicolumn{2}{c}{ CC EOS } \\
\hline$\Gamma_{1}$ & 0.25 & $\Gamma_{2}$ & 2 \\
$\rho_{1}^{r e f}\left(\mathrm{~kg} \cdot \mathrm{m}^{-3}\right)$ & 1840 & $\rho_{2}^{r e f}\left(\mathrm{~kg} . \mathrm{m}^{-3}\right)$ & 8900 \\
$A(\mathrm{~Pa})$ & $8.545 \times 10^{11}$ & $A_{1}(\mathrm{~Pa})$ & $1.45667 \times 10^{11}$ \\
$B(\mathrm{~Pa})$ & $2.05 \times 10^{10}$ & $A_{2}(\mathrm{~Pa})$ & $1.47751 \times 10^{11}$ \\
$R_{1}$ & 4.6 & $E_{1}$ & 2.994 \\
$R_{2}$ & 1.35 & $E_{2}$ & 1.994 \\
$e_{C J}\left(\mathrm{~J} . \mathrm{kg}^{-1}\right)$ & $2.610793 \times 10^{6}$ & $e_{0}\left({\left.\mathrm{~J} . \mathrm{kg}^{-1}\right)}\right.$ & $1.17900 \times 10^{5}$ \\
$p_{C J}(\mathrm{~Pa})$ & $37 \times 10^{9}$ & & \\
$\rho_{C J}\left(\mathrm{~kg} . \mathrm{m}^{-3}\right)$ & 2485.37 & & \\
\hline
\end{tabular}

Table 5: Initial conditions for Test 7

\begin{tabular}{llccccccc}
\hline & & $\boldsymbol{\alpha}_{\mathbf{2}}$ & $\begin{array}{c}\boldsymbol{\rho}_{\mathbf{1}} \\
\left(\mathrm{kg} . \mathrm{m}^{-3}\right)\end{array}$ & $\begin{array}{c}\boldsymbol{u}_{\mathbf{1}} \\
\left(\mathrm{m}_{\mathrm{s}}{ }^{-1}\right)\end{array}$ & $\begin{array}{c}\boldsymbol{p}_{\mathbf{1}} \\
(\mathrm{Pa})\end{array}$ & $\begin{array}{c}\boldsymbol{\rho}_{\mathbf{2}} \\
\left(\mathrm{kg} . \mathrm{m}^{-3}\right)\end{array}$ & $\begin{array}{c}\boldsymbol{u}_{\mathbf{2}} \\
\left(\mathrm{m} . \mathrm{s}^{-1}\right)\end{array}$ & $\begin{array}{c}\boldsymbol{p}_{\mathbf{2}} \\
(\mathrm{Pa})\end{array}$ \\
\hline \multirow{2}{*}{ Test $7[40]$} & Left state & $10^{-6}$ & 2485.37 & 0. & $37 \times 10^{9}$ & 8924 & 0. & 0. \\
& Right state & $1-10^{-6}$ & 2485.37 & 0. & $37 \times 10^{9}$ & 8924 & 0. & 0. \\
\hline
\end{tabular}



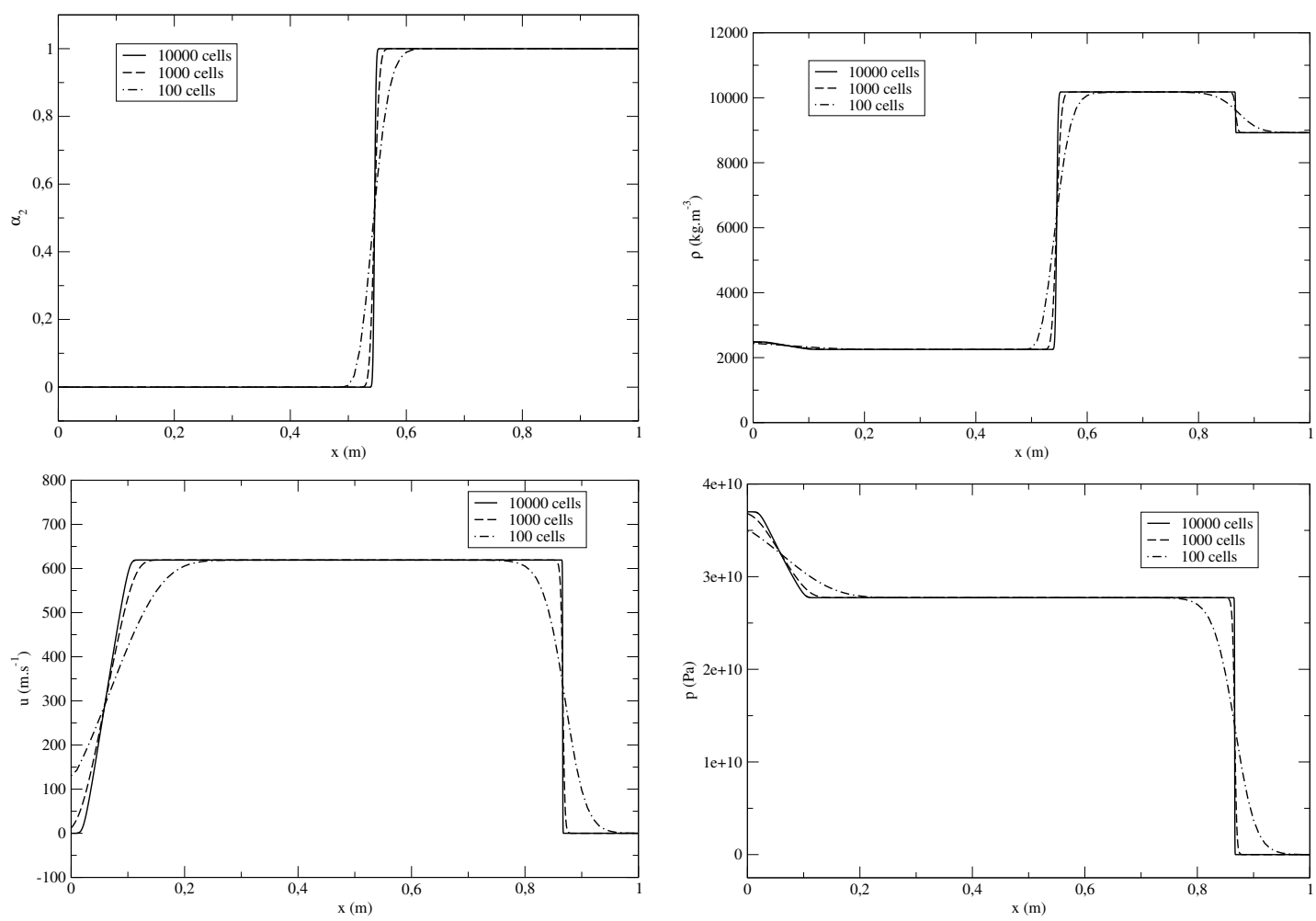

Figure 12: Numerical solutions of Test 7 obtained with the HLLAC scheme on different meshes at time $t_{f}=73 \times 10^{-6} s$. From the top left to the bottom right: $\alpha_{2}, \rho, u, p$ vs $x$. 


\section{$4.22 \mathrm{D}$ test-cases}

Three 2D test-cases have been selected to push forward the assessment of the present HLLAC scheme and compare it with the HLLC and the Rusanov schemes. The efficiency of the three schemes is also briefly examined here.

\subsubsection{Shock-bubble interaction}

The first 2D test studies the interaction between a shock wave and a Helium bubble. It has been experimentally investigated by Haas and Sturtevant [26] and widely used to assess models and numerical methods since then [15, 32, 33. A planar shock wave with $M=1.22$ is moving in the air towards a cylindrical Helium bubble. More precisely, the computational domain is $267 \mathrm{~mm} \times 89 \mathrm{~mm}$ with the initial shock located at $15 \mathrm{~mm}$ from the inlet, the radius of bubble is $25 \mathrm{~mm}$ and its center is vertically centered at $84.33 \mathrm{~mm}$ from the inlet of the domain. The computations are performed on a mesh made of $630 \times 210$ cells using transmissive inlet and outlet boundary conditions with walls at top and bottom. The two gases, Helium and air, are modeled as ideal gases $\left(p_{k}^{\infty}=0 \mathrm{~Pa}\right)$ with $\gamma_{1}=1.648$ and $\gamma_{2}=1.4$ respectively. The initial conditions of the test are presented in table 6 and the Courant number is set to $C_{C F L}=0.5$.

Figure 13 shows the comparison between numerical results of the three different schemes on the mean density $\rho=\alpha_{1} \rho_{1}+\alpha_{2} \rho_{2}$ at different times. As in many of the 1D Riemann problems, the results of the HLLAC and the HLLC schemes are similar. In contrast, the Rusanov scheme is very diffusive as it can be seen on the stationary interface of the bubble before being reached by the shock wave. This diffusion prevents the Rusanov scheme from capturing important physical phenomena in the interaction unlike the other schemes. Thus, we observe with the two HLLC-type schemes that the refracted shock wave in the bubble is ahead the shock due to the higher speed of sound in Helium. Moreover, a transmitted shock wave appears at the location where the reflected rarefaction wave meets the interface and it forms a $\lambda$-shock by interacting with the incoming shock. We also retrieve the beginning of the Richtmyer-Meshkov instability due to the interaction between the shock wave and the curved interface of the bubble. Finally we compare the CPU time of the computations of $1 \mathrm{~ms}$ with the three different schemes. Although, they provide very different results the Rusanov and the HLLAC schemes have a similar CPU cost whereas the HLLC scheme is 2.8 times slower on this test.

Table 6: Initial conditions for the shock-bubble interaction test-case

\begin{tabular}{lccccccc}
\hline & $\boldsymbol{\alpha}_{\mathbf{1}}$ & $\begin{array}{c}\boldsymbol{\rho}_{\mathbf{1}} \\
\left(\mathrm{kg} . \mathrm{m}^{-3}\right)\end{array}$ & $\begin{array}{c}\boldsymbol{u}_{\mathbf{1}} \\
\left(\mathrm{m} . \mathrm{s}^{-1}\right)\end{array}$ & $\begin{array}{c}\boldsymbol{p}_{\mathbf{1}} \\
(\mathrm{Pa})\end{array}$ & $\begin{array}{c}\boldsymbol{\rho}_{\mathbf{2}} \\
\left(\mathrm{kg} . \mathrm{m}^{-3}\right)\end{array}$ & $\begin{array}{c}\boldsymbol{u}_{\mathbf{2}} \\
\left(\mathrm{m}_{\mathrm{s}} \mathrm{s}^{-1}\right)\end{array}$ & $\begin{array}{c}\boldsymbol{p}_{\mathbf{2}} \\
(\mathrm{Pa})\end{array}$ \\
\hline Right state & $10^{-6}$ & 0.2546 & -114.42 & $10^{5}$ & 1.92691 & -114.42 & $1.5698 \times 10^{5}$ \\
Left state & $10^{-6}$ & 0.2546 & 0. & $10^{5}$ & 1.4 & 0. & $10^{5}$ \\
Bubble & $1-10^{-6}$ & 0.2546 & 0. & $10^{5}$ & 1.4 & 0. & $10^{5}$ \\
\hline
\end{tabular}

\subsubsection{Underwater explosion}

The second 2D test is taken from [24, 32] and focuses on an underwater explosion. A rectangular domain $[-2 ; 2] \mathrm{m} \times[-1.5 ; 1] \mathrm{m}$ with wall boundary conditions is considered. Initially, a circular bubble of high-pressure gas is located in the water under the air-water interface located at $y=0$. The radius of the bubble is $0.12 \mathrm{~m}$ and its center is located at $(0 ;-0.3) \mathrm{m}$. The air above the interface and the gas in the bubble are modeled as an ideal gas $\left(p_{1}^{\infty}=0 \mathrm{~Pa}\right)$ with $\gamma_{1}=1.4$. The Stiffened Gas EOS is used for the water $\left(p_{2}^{\infty}=6 \times 10^{8} \mathrm{~Pa}\right.$ and $\left.\gamma_{2}=4.4\right)$. Table 7 provides the initial conditions of the computations. This test is particularly suited to assess the robustness of the schemes due to the presence of a strong shock wave and material interfaces between vanishing phases. The initial pressure and density ratios are particularly high: around $10^{4}$ for pressure and $10^{3}$ for density. We use a grid with $400 \times 250$ cells and a Courant number $C_{C F L}=0.5$. Moreover the pressure and velocity 

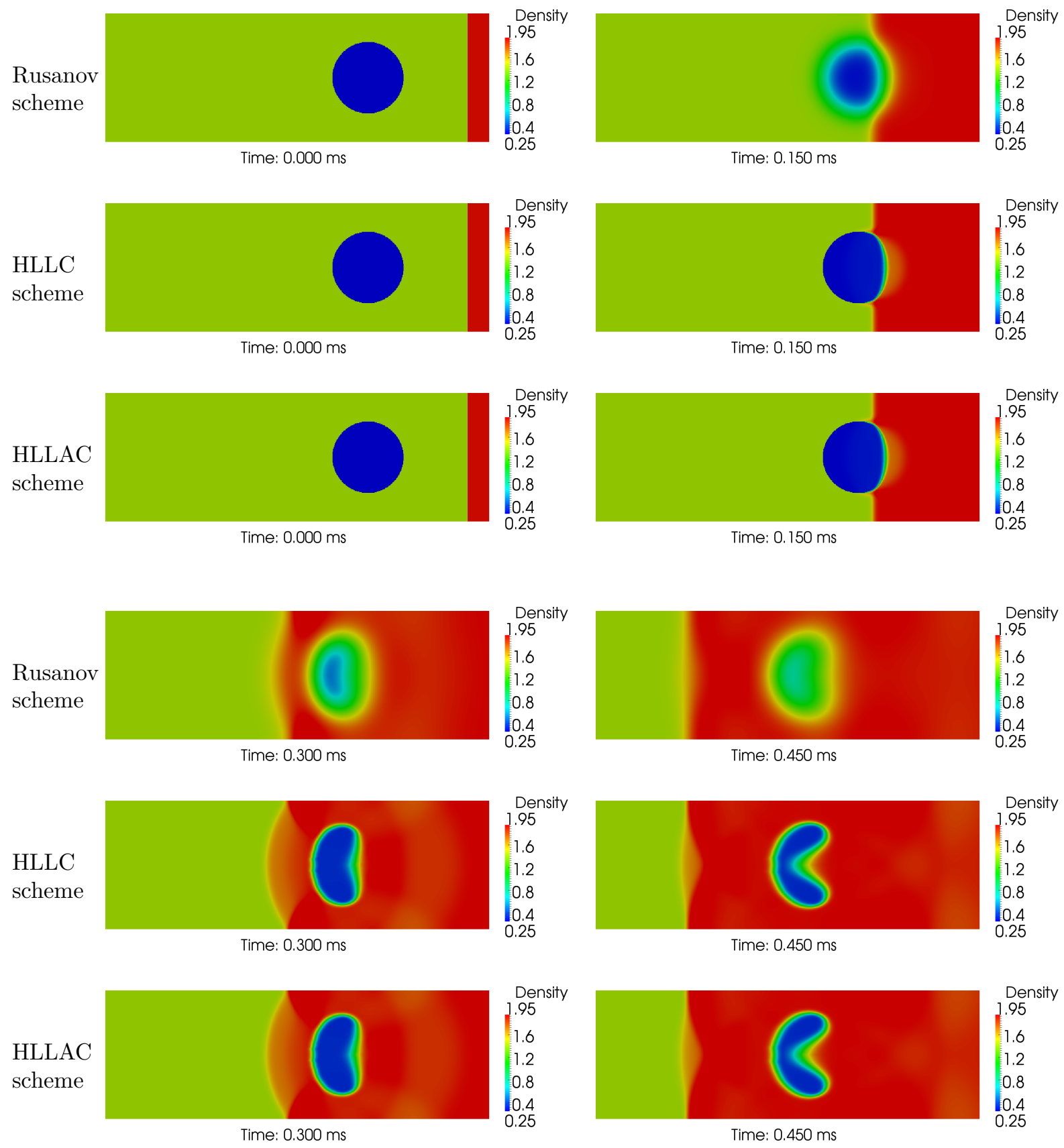

Figure 13: Shock and air/He bubble interaction: comparison between numerical solutions obtained with the Rusanov, the HLLC and the HLLAC schemes at different times. 
relaxation terms [29, 12, 36] are also used in this test-case. Details on those source terms can be found in appendix B. The time scales associated to those phenomena are set to very small values in order to obtain quasi-instantaneous relaxations: $\tau_{p}=10^{-12} \mathrm{~s}$ and $\tau_{u}=10^{-12} \mathrm{~s}$.

Figure 14 provides the numerical results of the computations with the different schemes on the mean density $\rho=\alpha_{1} \rho_{1}+\alpha_{2} \rho_{2}$ at several times. First, the HLLC scheme was not robust enough for this severe test. As in test 5, the HLLC does not preserve the positivity of the pressures of the intermediate states probably due to the presence of vanishing phases. Once again, the results obtained with the Rusanov and the HLLAC schemes look very different due to the inner numerical diffusion of the Rusanov scheme. Indeed, the initial pressure difference between the bubble and the water generates a shock wave in the water and a rarefaction wave in the bubble. Then, the shock wave reaches the free surface resulting in a reflected rarefaction wave in the water and a transmitted shock wave in the air which is much weaker and cannot be observed on the present density results. In the case of the Rusanov scheme, the horizontal air-water interface is very smeared. As it can be observed on the results obtained with the HLLAC scheme, the shape of the growing bubble evolves from a circular one to an oval one. More details on the physical phenomena can be found in [35]. The results obtained with the Rusanov scheme show a very different shape of the bubble due to its poor accuracy. Finally we note that the CPU cost of the HLLAC scheme is only $15 \%$ higher than the one of the Rusanov scheme on this test demonstrating the efficiency of the proposed scheme.

Table 7: Initial conditions for the underwater explosion test-case

\begin{tabular}{lccccccc}
\hline & $\boldsymbol{\alpha}_{\mathbf{1}}$ & $\begin{array}{c}\boldsymbol{\rho}_{\mathbf{1}} \\
\left(\mathrm{kg} \cdot \mathrm{m}^{-3}\right)\end{array}$ & $\begin{array}{c}\boldsymbol{u}_{\mathbf{1}} \\
\left(\mathrm{m} \cdot \mathrm{s}^{-1}\right)\end{array}$ & $\begin{array}{c}\boldsymbol{p}_{\mathbf{1}} \\
\mathrm{Pa})\end{array}$ & $\begin{array}{c}\boldsymbol{\rho}_{\mathbf{2}} \\
\left(\mathrm{kg} \cdot \mathrm{m}^{-3}\right)\end{array}$ & $\begin{array}{c}\boldsymbol{u}_{\mathbf{2}} \\
\left(\mathrm{m} . \mathrm{s}^{-1}\right)\end{array}$ & $\begin{array}{c}\boldsymbol{p}_{\mathbf{2}} \\
(\mathrm{Pa})\end{array}$ \\
\hline Water & $10^{-6}$ & 1.225 & 0. & $1.01325 \times 10^{5}$ & 1000 & 0. & $1.01325 \times 10^{5}$ \\
Air & $1-10^{-6}$ & 1.225 & 0. & $1.01325 \times 10^{5}$ & 1000 & 0. & $1.01325 \times 10^{5}$ \\
Gas bubble & $1-10^{-6}$ & 1250 & 0. & $10^{9}$ & 1000 & 0. & $10^{9}$ \\
\hline
\end{tabular}

\subsubsection{Cylindrical underwater explosion near a planar wall}

The third $2 \mathrm{D}$ test is similar to the previous one except that a rigid planar wall replaces the free surface. Therefore the strong interaction between a shock wave in the water and a planar wall can be investigated. This test-case was previously studied in 44 , 37. A rectangular computational domain $[-6 ; 6]$ $\mathrm{m} \times[-6 ; 3] \mathrm{m}$ is considered. The upper boundary condition located at $y=3 \mathrm{~m}$ is a rigid wall whereas the other boundary conditions are transmissive. Initially, a 1-m radius explosive gas bubble is located at $(0 ; 0)$. This gas is modeled as an ideal gas $\left(p_{1}^{\infty}=0 \mathrm{~Pa}\right)$ with $\gamma_{1}=2$ whereas the water is modeled as a Stiffened Gas with $p_{2}^{\infty}=3 \times 10^{8} \mathrm{~Pa}$ and $\gamma_{2}=7.15$. Table 8 provides the initial conditions used for the present computations. As in [37, a small amount of gas with $\alpha_{1}=0.005$ is uniformly distributed in the water. A very fine mesh is used with $1200 \times 900$ cells. All of the computations are performed with a Courant number $C_{C F L}=0.5$. As in the previous underwater explosion test, the pressure and velocity relaxation terms [29, 12, 36] are used with $\tau_{p}=10^{-12} \mathrm{~s}$ and $\tau_{u}=10^{-12} \mathrm{~s}$ (see appendix B for details on those source terms).

Table 8: Initial conditions for the computation of the 2D cylindrical underwater explosion near a planar wall

\begin{tabular}{lccccccc}
\hline & $\boldsymbol{\alpha}_{\mathbf{1}}$ & $\begin{array}{c}\boldsymbol{\rho}_{\mathbf{1}} \\
\left(\mathrm{kg} \cdot \mathrm{m}^{-3}\right)\end{array}$ & $\begin{array}{c}\boldsymbol{u}_{\mathbf{1}} \\
\left({\left.\mathrm{m} . \mathrm{s}^{-1}\right)}\right.\end{array}$ & $\begin{array}{c}\boldsymbol{p}_{\mathbf{1}} \\
(\mathrm{Pa})\end{array}$ & $\begin{array}{c}\boldsymbol{\rho}_{\mathbf{2}} \\
\left(\mathrm{kg} \cdot \mathrm{m}^{-3}\right)\end{array}$ & $\begin{array}{c}\boldsymbol{u}_{\mathbf{2}} \\
\left(\mathrm{m}_{\mathrm{s}} \mathrm{s}^{-1}\right)\end{array}$ & $\begin{array}{c}\boldsymbol{p}_{\mathbf{2}} \\
(\mathrm{Pa})\end{array}$ \\
\hline Water & 0.005 & 1.27 & 0. & $10^{5}$ & 1000 & 0. & $10^{5}$ \\
Gas bubble & 0.995 & 1270 & 0. & $8.29 \times 10^{8}$ & 1000 & 0. & $8.29 \times 10^{8}$ \\
\hline
\end{tabular}

Figure 15 provides the numerical results of the computation with the different schemes on the mean 


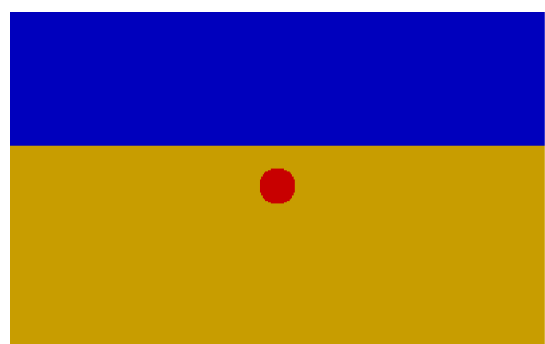

Time: $0.00 \mathrm{~ms}$

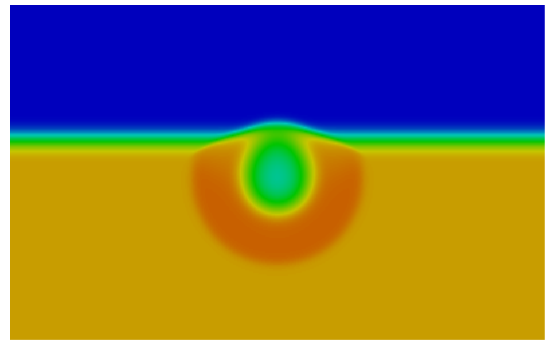

Time: $0.25 \mathrm{~ms}$

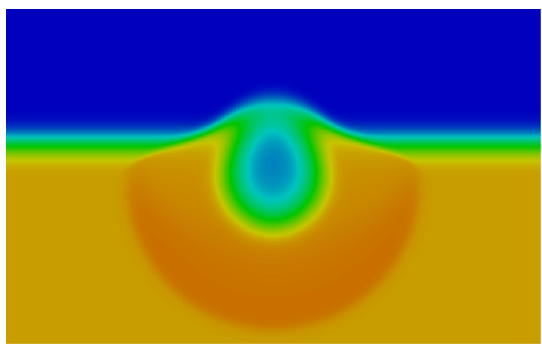

Time: $0.50 \mathrm{~ms}$

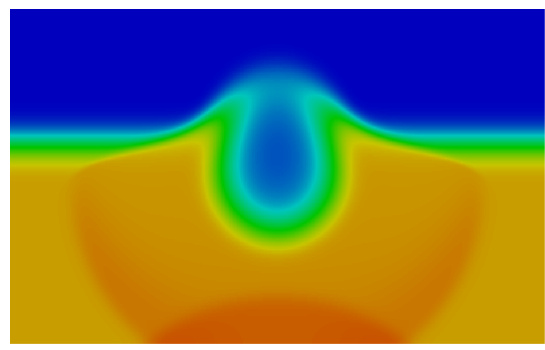

Time: $0.75 \mathrm{~ms}$

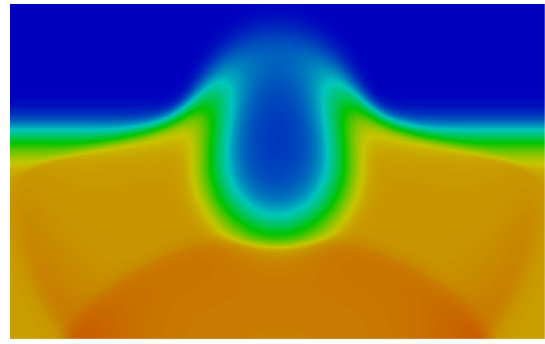

Time: $1.00 \mathrm{~ms}$

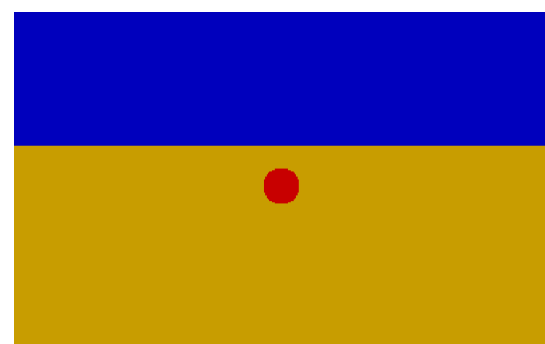

Time: $0.00 \mathrm{~ms}$

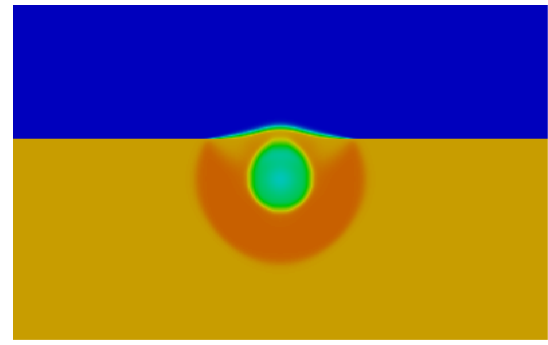

Time: $0.25 \mathrm{~ms}$

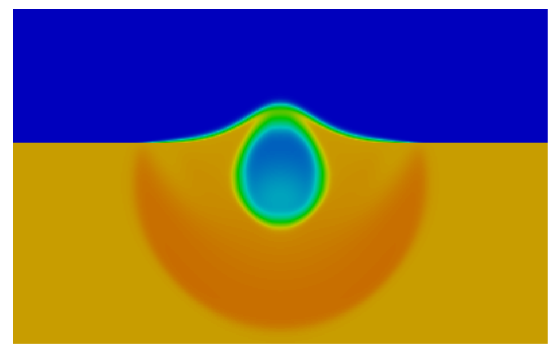

Time: $0.50 \mathrm{~ms}$

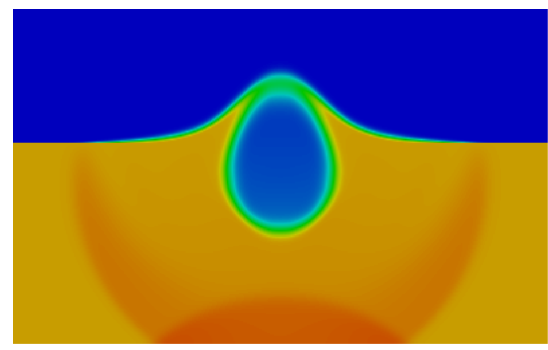

Time: $0.75 \mathrm{~ms}$

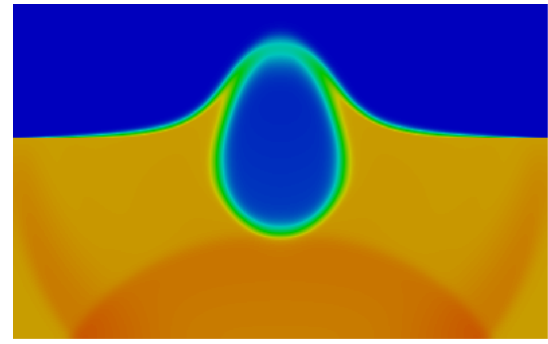

Time: $1.00 \mathrm{~ms}$

Figure 14: Underwater explosion: comparison between numerical solutions obtained with the Rusanov scheme (left column) and the HLLAC scheme (right column) at different times. 
density $\left(\rho=\alpha_{1} \rho_{1}+\alpha_{2} \rho_{2}\right)$ fields in conjunction with the mean pressure $\left(p=\alpha_{1} p_{1}+\alpha_{2} p_{2}\right)$ contours at different time instances. First, as in the previous underwater explosion test, the HLLC scheme is not robust enough on this challenging test. At $t=1.5 \mathrm{~ms}$, the main shock generated by the explosion has been reflected from the upper planar rigid wall (see Figure 15-(a)). The excessive numerical diffusion of the Rusanov scheme smears both the density jump located at the gas-water interface and the shock waves which propagate in the water. Then, the reflected shock wave interacts with the gas-water interface resulting in a rarefaction wave propagating in the water towards the upper wall and a weak shock wave propagating in the gas bubble (with a lower celerity) which are clearly visible at $t=2 \mathrm{~ms}$ (see Figure 15-(b)). Notice that the rarefaction wave is strongly affected by the numerical dissipation of the Rusanov scheme. As a consequence, the obtained reflection of this rarefaction wave at the upper wall is weaker with the use of the Rusanov scheme than with the use of the HLLAC scheme. Due to this reflection, a low pressure region is generated near the wall leading to an inception of cavitation. Notice that the cavitation is only obtained with the HLLAC scheme. Afterward, the cavitation pocket collapses as it can be seen on the numerical results obtained with the HLLAC scheme. Figure 15-(e) shows that, at $t=5.5 \mathrm{~ms}$, the cavitation region near the center of the wall has collapsed whereas cavitation regions still exist on both sides of the collapse zone.

The pressure and the gas volume fraction histories at the center of the wall are presented on Figure 16. All of the computations retrieve the main pressure peak at $t=1 \mathrm{~ms}$ which corresponds to the reflection of the main shock wave at the upper wall. The time of occurrence and the shape of this peak are in agreement with the previous computations performed by Xie et al. [44. (see Figure 16 page 1190) and by Ma et al. 37] (see Figure 13 page 16). The maximal amplitude of the pressure peak (6600 bar with HLLAC and 6400 bar with Rusanov) is very close to the Xie's solution (6500 bar). The generation of cavitation at $t=3.7 \mathrm{~ms}$ is only retrieved with the the HLLAC scheme. In contrast, no cavitation occurs during the computation using the Rusanov scheme as it can also be noticed on the time evolution of the gas volume fraction. However, the collapse of the cavitation pocket is predicted earlier in the present computation $(t=4.55 \mathrm{~ms})$ in comparison with the Xie's solution $(t=5.05 \mathrm{~ms})$ and the Ma's solution $(t=5.25 \mathrm{~ms})$. This discrepancy may be explained by the use of first-order schemes in the present computations in contrast to the previous ones [44, 37. In the same way, the earliest stages of the gas volume fraction history obtained with the HLLAC scheme are in agreement with the Ma's solution (see Figure 14 page 16), i.e. strong drop at $t=1 \mathrm{~ms}$ when the shock wave reflects at the wall and rapid increase at $t=3.7 \mathrm{~ms}$ when cavitation occurs. However, the gas volume fraction strongly reduces at $t=4.55 \mathrm{~ms}$ in the present computation which corresponds to the collapse of the cavitation pocket. Besides the maximal value of the gas volume fraction is also lower in the present computation in comparison to the Ma's computation (0.0165 vs 0.04-0.05). In order to investigate the influence of the numerical errors induced by the use of first-order schemes, a finer mesh $2400 \times 1800$ cells is also considered to reduce the corresponding numerical diffusion errors. The associated results show that the collapse of the cavitation pocket is postponed $(t=4.7 \mathrm{~ms})$ and the maximum value of $\alpha_{1}$ is increased (0.04). 
(a)
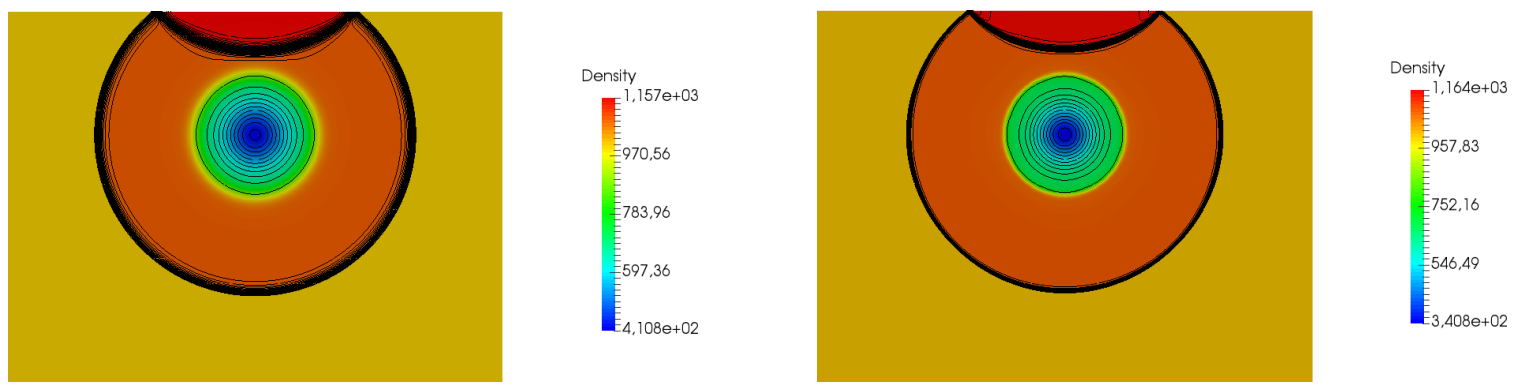

(b)
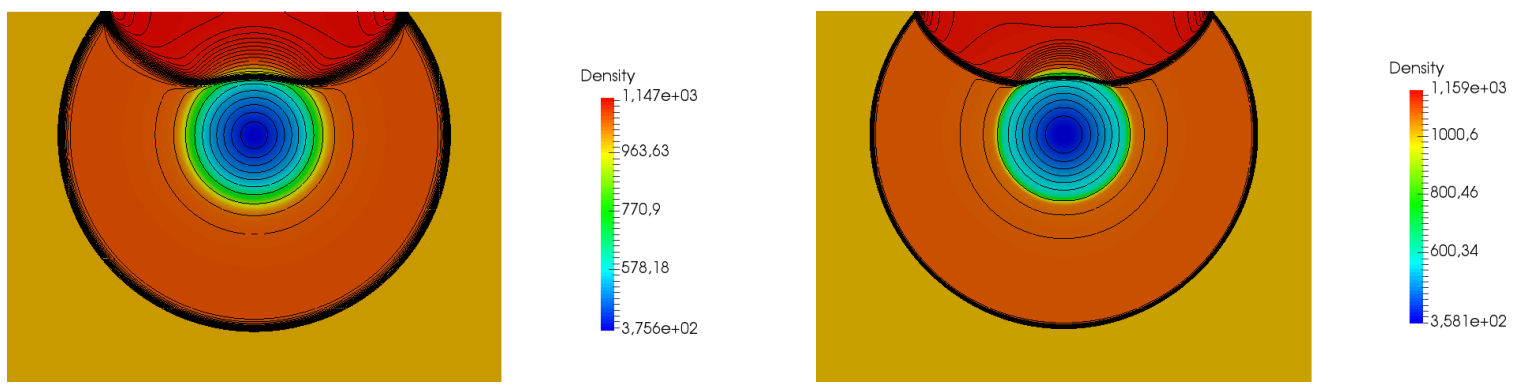

(c)
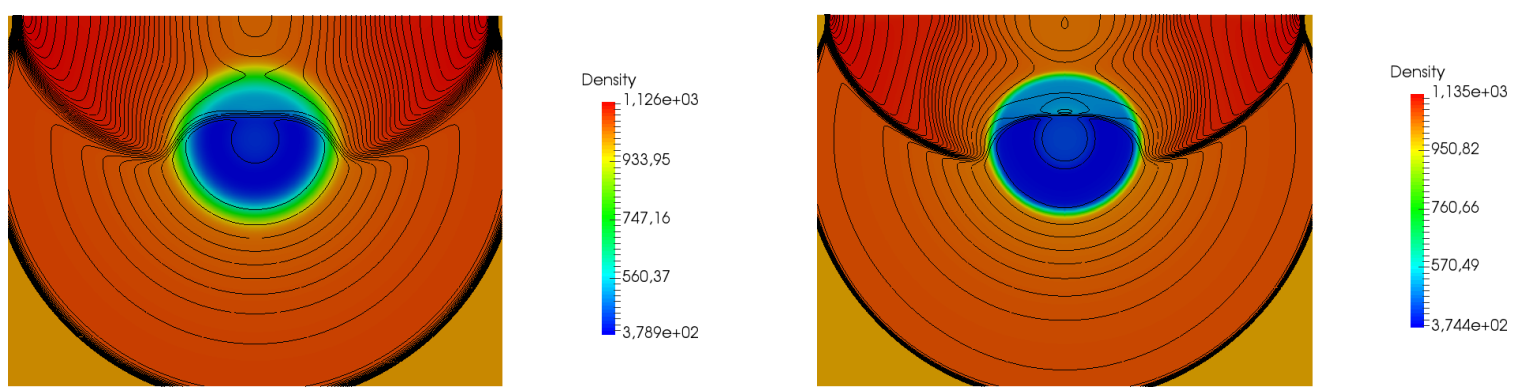

(d)
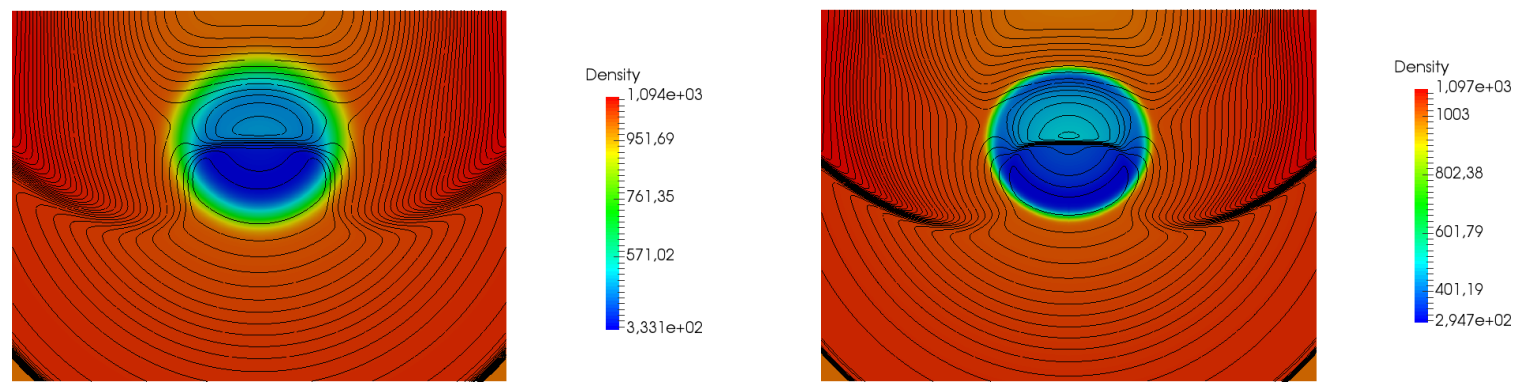

(e)
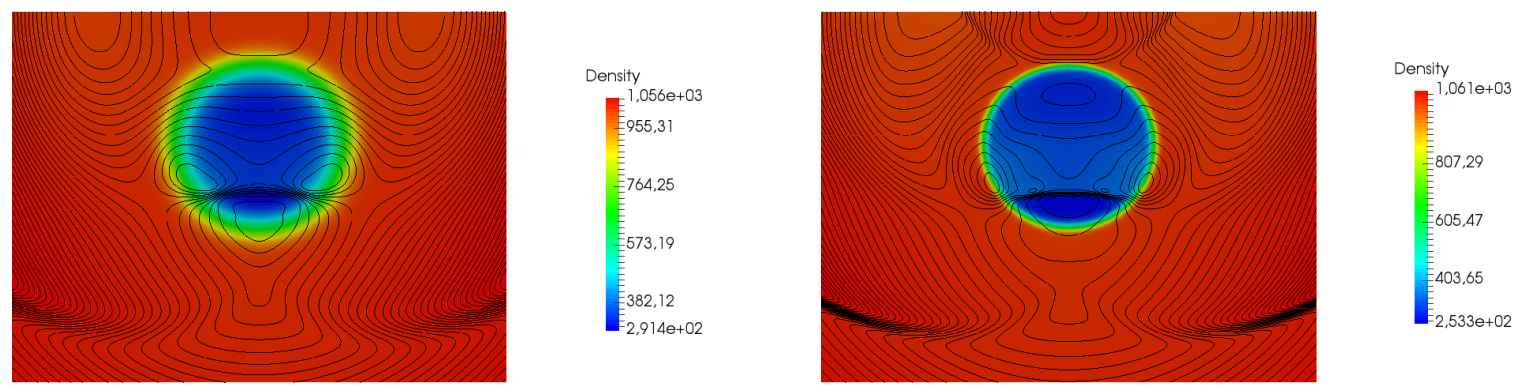

Figure 15: Cylindrical underwater explosion near a planar wall: comparison between numerical solutions obtained with the Rusanov (left) and HLLAC (right) schemes at different instants: (a) $t=1.5$ $\mathrm{ms}$, (b) $t=2 \mathrm{~ms}$, (c) $t=3 \mathrm{~ms}$, (d) $t=4 \mathrm{~ms}$, and (e) $t=5.5 \mathrm{~ms}$. 
(a)

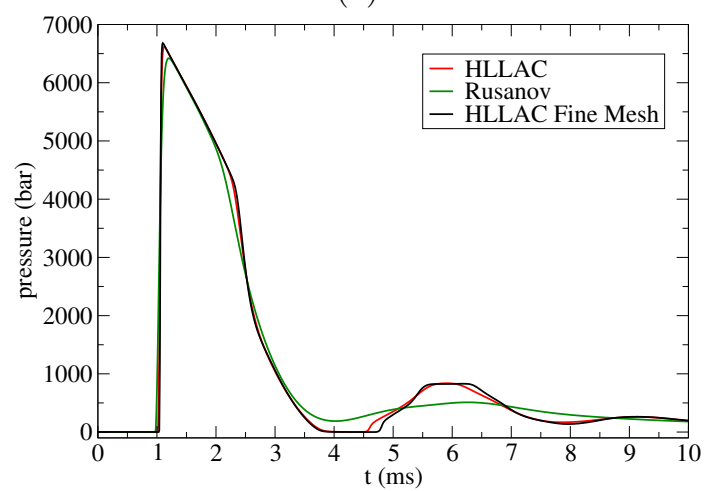

(b)

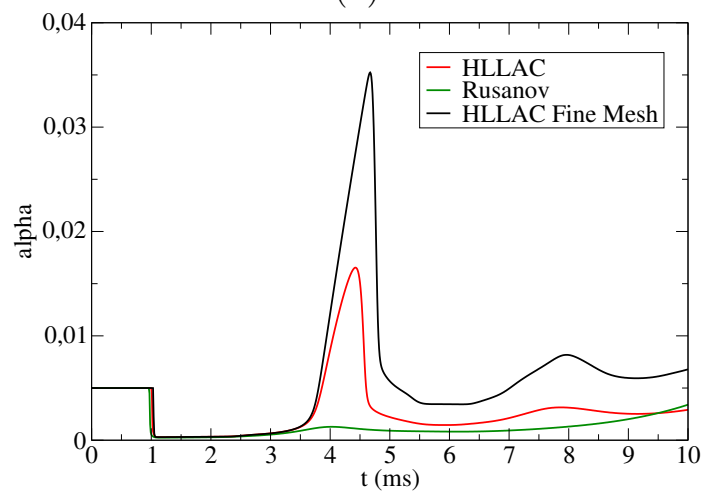

Figure 16: Cylindrical underwater explosion near a planar wall: comparison of the time evolutions of the pressure (a) and the gas volume fraction (b) obtained with the HLLAC and Rusanov schemes.

\section{Conclusion}

Based on the solver proposed by Tokareva and Toro [42], a new HLLC-type Riemann scheme for the computation of the Baer-Nunziato model has been proposed. The key idea of that new scheme is an approximation of the two-phase contact of the model based on the Riemann invariants of this wave. It is only valid for "subsonic" flows but physically-relevant in those cases. An important consequence of the approximation is that the solver can directly deal with any Equation Of State. Moreover the solver no longer requires the resolution of a non-linear equation using an iterative procedure since the intermediate states are obtained as the solution of a linear system unlike the original one. Finally, the approximation of the two-phase contact is in agreement with the thin layer approximation proposed by Schwendeman et al. [41] and therefore a similar numerical treatment of the non-conservative products is used. The proposed solver has been compared with the original one and the Rusanov scheme on both 1D and 2D test-cases. Several 1D Riemann problems have been selected in order to assess the accuracy and the robustness of the scheme. The last 1D Riemann problem also demonstrates its ability to deal with complex Equations Of State. Grid convergence studies have been carried out and particular attention has been paid to the case of vanishing phases. Numerical results show that the original HLLC scheme is the most accurate one but the new HLLC-type scheme provides similar results in most cases and appears to be more robust on challenging tests. An efficiency study has also been carried out showing that the proposed scheme is more efficient than the original HLLC one. Three 2D test-cases have then been studied, a shock-bubble interaction and two underwater explosions which are severe test-cases with violent shock waves and material interfaces. Once again, the new scheme exhibits accuracy and robustness. Moreover, it appears to be very efficient since its computational cost is similar to the Rusanov one on those tests whereas it is more accurate. Thus, the proposed scheme appears to be an excellent candidate for the computation of more realistic situations since it is a good compromise between the accuracy of the original HLLC scheme and the robustness of the Rusanov scheme, while showing great efficiency.

Only first-order schemes have been considered in the present work but the extension of the proposed solver to the second-order, using the approach of Schwendeman et al. [41] for example, should be assessed. Based on previous work [15, the solver should also be tested in the ALE framework, particularly its robustness, in order to tackle the computations of two-phase flows with fluid-structures interactions.

Acknowledgements The first author received a financial support through the EDF-CIFRE contract 0459-2013. Computational facilities were provided by EDF. Numerical simulations have been performed with the Europlexus software. 


\section{Appendix A. Connection through the two-phase contact}

This appendix is dedicated to the connection through the two-phase contact between two states $\mathbf{W}_{l}$ and $\mathbf{W}_{r}$ where we use the following notation:

$$
\mathbf{W}_{K}=\left(\begin{array}{c}
\left(\alpha_{1}\right)_{K} \\
\left(\rho_{1}\right)_{K} \\
\left(u_{1}\right)_{K} \\
\left(p_{1}\right)_{K} \\
\left(\rho_{2}\right)_{K} \\
\left(u_{2}\right)_{K} \\
\left(p_{2}\right)_{K}
\end{array}\right) \quad, \quad K=l, r
$$

More precisely, we assume $\mathbf{W}_{l},\left(\alpha_{1}\right)_{r},\left(\rho_{2}\right)_{r}$ and look for $\mathbf{W}_{r}$ such that:

$$
I_{k}\left(\mathbf{W}_{r}\right)=I_{k}\left(\mathbf{W}_{l}\right) \quad, \quad k=1,5
$$

where $\left(I_{k}\right)_{k=1,5}$ are the exact Riemann invariants of the two-phase contact given in equation (9). To that end, we first define the main unknown $x=\left(\rho_{1}\right)_{r}$ and recast the preservation of $I_{4}$ in the following way:

$$
I_{4}\left(\mathbf{W}_{l}\right)=h_{1}\left(x,\left(p_{1}\right)_{r}\right)+\frac{1}{2}\left(\frac{I_{2}\left(\mathbf{W}_{l}\right)}{\left(\alpha_{1}\right)_{r} x}\right)^{2}
$$

where $h_{1}\left(\rho_{1}, p_{1}\right)$ is the specific internal enthalpy of phase 1 . We also define the function $\mathcal{P}_{1}$ such that $p_{1}=\mathcal{P}_{1}\left(\rho_{1}, s_{1}\right)$. Thus, equation 29 can be written as a non-linear equation on $x$ :

$$
f(x)=I_{4}\left(\mathbf{W}_{l}\right) \quad \text { with } \quad f(x)=h_{1}\left(x, \mathcal{P}_{1}\left(x, I_{5}\left(\mathbf{W}_{l}\right)\right)\right)+\frac{1}{2}\left(\frac{I_{2}\left(\mathbf{W}_{l}\right)}{\left(\alpha_{1}\right)_{r} x}\right)^{2}
$$

Once this equation has been solved, $W_{r}$ can easily be fully determined. Indeed, $\left(p_{1}\right)_{r}=\mathcal{P}_{1}\left(x, I_{5}\left(\mathbf{W}_{l}\right)\right)$, $\left(u_{1}\right)_{r}$ and $\left(u_{2}\right)_{r}$ are obtained thanks to the preservation of $I_{1}$ and $I_{2}$. Finally the last Riemann invariant $I_{3}$ provides us the remaining unknown $\left(p_{2}\right)_{r}$. Thus the connection through the two-phase contact only depends on the resolution of the non-linear equation 30 .

For classical EOS, the first term of $f(x)$ is an increasing function of $x$. For example, the ideal gas EOS gives:

$$
h_{1}\left(x, \mathcal{P}_{1}\left(x, I_{5}\left(\mathbf{W}_{l}\right)\right)\right)=\frac{\gamma_{1} I_{5}\left(\mathbf{W}_{l}\right)}{\gamma_{1}-1} x^{\gamma_{1}-1}
$$

Therefore, for non-zero values of $I_{2}\left(\mathbf{W}_{l}\right)$, the function $f(x)$ admits a minimum for $x=x_{0}$. Moreover $f(x)$ is decreasing for $x$ in $\left.] 0, x_{0}\right]$ and then increasing for $x$ in $\left[x_{0},+\infty[\right.$. As a consequence, the equation (30) has no solution if $f\left(x_{0}\right)>I_{4}\left(\mathbf{W}_{l}\right)$, one solution if $f\left(x_{0}\right)=I_{4}\left(\mathbf{W}_{l}\right)$ and two solutions if $f\left(x_{0}\right)<I_{4}\left(\mathbf{W}_{l}\right)$ which corresponds to figure 17. It must be underlined that, when $f\left(x_{0}\right)>I_{4}\left(\mathbf{W}_{l}\right)$, no state $\mathbf{W}_{r}$ can be connected to $\mathbf{W}_{l}$ by the two-phase contact. 


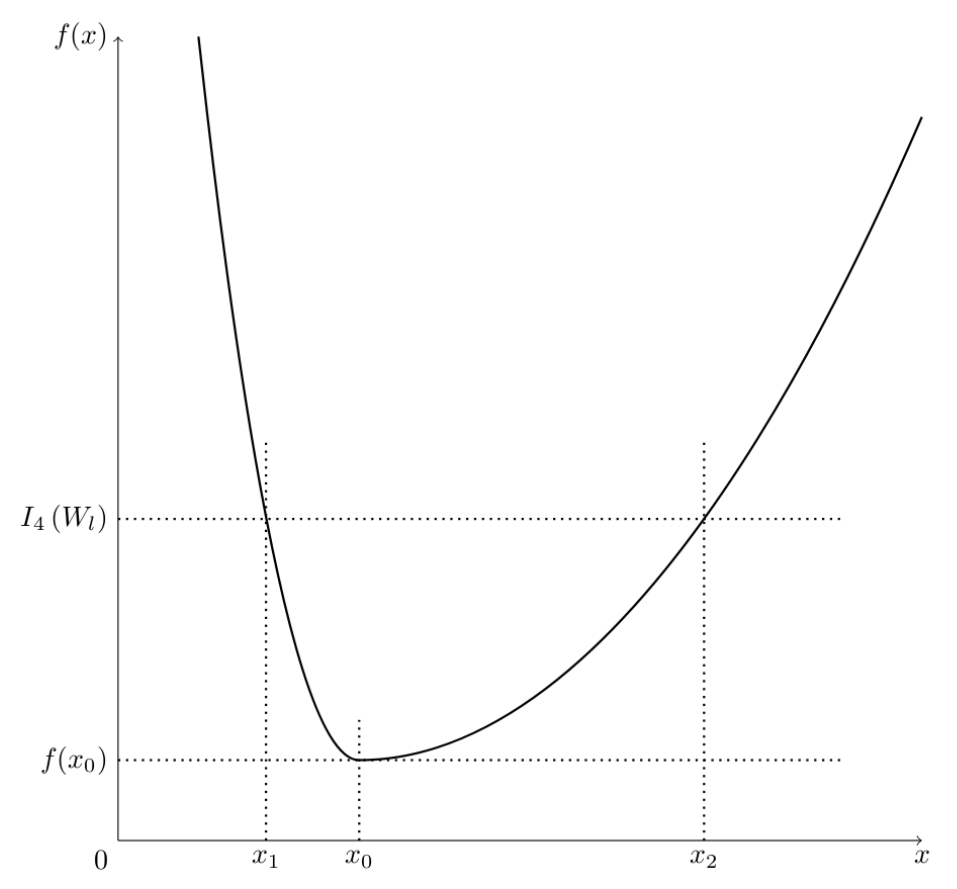

Figure 17: Resolution of equation 30

\section{Appendix B. Pressure and velocity relaxations}

The Baer-Nunziato model with the source terms including pressure and velocity relaxations can be written as follows:

$$
\left\{\begin{array}{lll}
\partial_{t}\left(\alpha_{1}\right) & +u_{I} \partial_{x}\left(\alpha_{1}\right) & =K_{p}\left(p_{1}-p_{2}\right) \\
\partial_{t}\left(\alpha_{k} \rho_{k}\right) & +\partial_{x}\left(\alpha_{k} \rho_{k} u_{k}\right) & =0 \\
\partial_{t}\left(\alpha_{k} \rho_{k} u_{k}\right) & +\partial_{x}\left(\alpha_{k} \rho_{k} u_{k}^{2}+\alpha_{k} p_{k}\right)+(-1)^{k} p_{I} \partial_{x}\left(\alpha_{1}\right) & =K_{u}\left(u_{j}-u_{k}\right) \\
\partial_{t}\left(\alpha_{k} \rho_{k} e_{k}\right) & +\partial_{x}\left(\alpha_{k} \rho_{k} e_{k} u_{k}+\alpha_{k} p_{k} u_{k}\right)+(-1)^{k+1} p_{I} \partial_{t}\left(\alpha_{1}\right) & =\frac{u_{1}+u_{2}}{2} K_{u}\left(u_{j}-u_{k}\right)
\end{array}\right.
$$

where $k=1,2$ and $j=3-k$. The scalars $K_{p}$ and $K_{u}$ are positive functions given by [29]:

$$
K_{p}=\frac{1}{\tau_{p}} \frac{\alpha_{1} \alpha_{2}}{\left|p_{1}\right|+\left|p_{2}\right|} \quad \text { and } \quad K_{u}=\frac{1}{\tau_{u}} \frac{\alpha_{1} \rho_{1} \alpha_{2} \rho_{2}}{\alpha_{1} \rho_{1}+\alpha_{2} \rho_{2}}
$$

where $\tau_{p}$ and $\tau_{u}$ are respectively the time scales of pressure and velocity relaxation. System (31) is approximated using a fractional step method proposed in [29. More details on the different relaxation substeps can be found in [29, 12, 36]. 


\section{References}

[1] Europlexus User's Manual. Technical Report, Joint Research Centre (JRC), Commissariat à l'énergie atomique et aux énergies alternatives (CEA), 2016.

URL http://europlexus.jrc.ec.europa.eu/public/manual_html/index.html

[2] A. Ambroso, C. Chalons, F. Coquel, and T. Galié. Relaxation and numerical approximation of a two-fluid two-pressure diphasic model. ESAIM: Mathematical Modelling and Numerical Analysis, 43(06):1063-1097, 2009.

[3] A. Ambroso, C. Chalons, and P.-A. Raviart. A Godunov-type method for the seven-equation model of compressible two-phase flow. Computers $\mathcal{E}$ Fluids, 54:67-91, 2012.

[4] N. Andrianov and G. Warnecke. The Riemann problem for the Baer-Nunziato two-phase flow model. Journal of Computational Physics, 195(2):434-464, 2004.

[5] M. Baer and J. Nunziato. A two-phase mixture theory for the deflagration-to-detonation transition (DDT) in reactive granular materials. International Journal of Multiphase Flow, 12(6):861-889, 1986.

[6] P. Batten, N. Clarke, C. Lambert, and D. Causon. On the Choice of Wavespeeds for the HLLC Riemann Solver. SIAM Journal on Scientific Computing, 18(6):1553-1570, 1997.

[7] A. Chinnayya, E. Daniel, and R. Saurel. Modelling detonation waves in heterogeneous energetic materials. Journal of Computational Physics, 196(2):490-538, 2004.

[8] F. Coquel, T. Gallouët, J.-M. Hérard, and N. Seguin. Closure laws for a two-fluid two-pressure model. Comptes Rendus Mathematique, 334(10):927-932, 2002.

[9] F. Coquel, J.-M. Hérard, K. Saleh, and N. Seguin. A robust entropy-satisfying finite volume scheme for the isentropic Baer-Nunziato model. ESAIM: Mathematical Modelling and Numerical Analysis, 48(1):165-206, 2014.

[10] F. Coquel, J.-M. Hérard, K. Saleh, and N. Seguin. A Positive and Entropy-Satisfying Finite Volume Scheme for the Baer-Nunziato Model, 2016. Preprint. URL https://hal .archives-ouvertes.fr/hal-01261458

[11] F. Crouzet, F. Daude, P. Galon, P. Helluy, J.-M. Hérard, O. Hurisse, and Y. Liu. Approximate solutions of the Baer-Nunziato Model. ESAIM: Proceedings, 40:63-82, 2013.

[12] F. Crouzet, F. Daude, P. Galon, J.-M. Hérard, O. Hurisse, and Y. Liu. Validation of a two-fluid model on unsteady liquid-vapor water flows. Computers \& Fluids, 119:131-142, 2015.

[13] G. Dal Maso, P. G. LeFloch, and F. Murat. Definition and weak stability of nonconservative products. Journal de mathématiques pures et appliquées, 74(6):483-548, 1995.

[14] S. Dallet. A comparative study of numerical schemes for the Baer-Nunziato model. submitted to International Journal On Finite Volumes, 2016.

[15] F. Daude and P. Galon. On the computation of the Baer-Nunziato model using ALE formulation with HLL- and HLLC-type solvers towards fluid-structure interactions. Journal of Computational Physics, 304:189-230, 2016.

[16] V. Deledicque and M. V. Papalexandris. An exact Riemann solver for compressible two-phase flow models containing non-conservative products. Journal of Computational Physics, 222(1):217-245, 2007.

[17] D. A. Drew and S. L. Passman. Theory of Multicomponent Fluids. Springer Verlag, 1999. 
[18] M. Dumbser and E. F. Toro. A Simple Extension of the Osher Riemann Solver to Non-conservative Hyperbolic Systems. Journal of Scientific Computing, 48(1-3):70-88, 2010.

[19] P. Embid and M. Baer. Mathematical analysis of a two-phase continuum mixture theory. Continuum Mechanics and Thermodynamics, 4(4):279-312, 1992.

[20] D. Furfaro and R. Saurel. A simple HLLC-type Riemann solver for compressible non-equilibrium two-phase flows. Computers \& Fluids, 111:159-178, 2015.

[21] T. Gallouët, J.-M. Hérard, and N. Seguin. Numerical Modeling Of Two-Phase Flows Using The Two-Fluid Two-Pressure Approach. Mathematical Models and Methods in Applied Sciences, 14(05):663-700, 2004.

[22] S. Gavrilyuk and R. Saurel. Mathematical and Numerical Modeling of Two-Phase Compressible Flows with Micro-Inertia. Journal of Computational Physics, 175(1):326-360, 2002.

[23] J. Glimm, D. Saltz, and D. H. Sharp. Renormalization group solution of two-phase flow equations for Rayleigh-Taylor mixing. Physics Letters A, 222(3):171-176, 1996.

[24] J. W. Grove and R. Menikoff. Anomalous reflection of a shock wave at a fluid interface. Journal of Fluid Mechanics, 219:313-336, 1990.

[25] V. Guillemaud. Modélisation et simulation numérique des écoulements diphasiques par une approche bifluide à deux pressions. Ph.D. thesis, Université de Provence - Aix-Marseille I, 2007. URL https://tel.archives-ouvertes.fr/tel-00169178/document

[26] J.-F. Haas and B. Sturtevant. Interaction of weak shock waves with cylindrical and spherical gas inhomogeneities. Journal of Fluid Mechanics, 181:41-76, 1987.

[27] A. Harten, P. Lax, and B. van Leer. On Upstream Differencing and Godunov-Type Schemes for Hyperbolic Conservation Laws. SIAM Review, 25(1):35-61, 1983.

[28] J.-M. Hérard. A three-phase flow model. Mathematical and Computer Modelling, 45(5-6):732-755, 2007.

[29] J.-M. Hérard and O. Hurisse. A fractional step method to compute a class of compressible gasliquid flows. Computers \& Fluids, 55:57-69, 2012.

[30] M. Ishii. Thermo-fluid dynamic theory of two-phase flows. Collection de la Direction des Etudes et Recherches d'Electricité de France, 1975.

[31] H. Jin, J. Glimm, and D. H. Sharp. Entropy of averaging for compressible two-pressure two-phase flow models. Physics Letters A, 360(1):114-121, 2006.

[32] S. Kokh and F. Lagoutière. An anti-diffusive numerical scheme for the simulation of interfaces between compressible fluids by means of a five-equation model. Journal of Computational Physics, 229(8):2773-2809, 2010.

[33] J. J. Kreeft and B. Koren. A new formulation of Kapila's five-equation model for compressible twofluid flow, and its numerical treatment. Journal of Computational Physics, 229(18):6220-6242, 2010.

[34] S. Liang, W. Liu, and L. Yuan. Solving seven-equation model for compressible two-phase flow using multiple GPUs. Computers \& Fluids, 99:156-171, 2014.

[35] T. G. Liu, B. C. Khoo, and K. S. Yeo. The simulation of compressible multi-medium flow: II. Applications to 2D underwater shock refraction. Computers \& Fluids, 30(3):315-337, 2001. 
[36] H. Lochon, F. Daude, P. Galon, and J.-M. Hérard. Comparison of two-fluid models on steam-water transients. ESAIM: Mathematical Modelling and Numerical Analysis, 2016.

[37] Z. Ma, D. Causon, L. Qian, H. Gu, C. Mingham, and P. M. Ferrer. A GPU based compressible multiphase hydrocode for modelling violent hydrodynamic impact problems. Computers $\&$ Fluids, 120:1-23, 2015.

[38] S. Müller, M. Hantke, and P. Richter. Closure conditions for non-equilibrium multi-component models. Continuum Mechanics and Thermodynamics, pages 1-33, 2015.

[39] V. H. Ransom and D. L. Hicks. Hyperbolic two-pressure models for two-phase flow. Journal of Computational Physics, 53(1):124-151, 1984.

[40] R. Saurel and R. Abgrall. A Multiphase Godunov Method for Compressible Multifluid and Multiphase Flows. Journal of Computational Physics, 150(2):425-467, 1999.

[41] D. W. Schwendeman, C. W. Wahle, and A. K. Kapila. The Riemann problem and a high-resolution Godunov method for a model of compressible two-phase flow. Journal of Computational Physics, 212(2):490-526, 2006.

[42] S. Tokareva and E. Toro. HLLC-type Riemann solver for the Baer-Nunziato equations of compressible two-phase flow. Journal of Computational Physics, 229(10):3573-3604, 2010.

[43] E. F. Toro, M. Spruce, and W. Speares. Restoration of the contact surface in the HLL-Riemann solver. Shock Waves, 4(1):25-34, 1994.

[44] W. Xie, T. Liu, and B. Khoo. Application of a one-fluid model for large scale homogeneous unsteady cavitation: The modified Schmidt model. Computers \& Fluids, 35(10):1177-1192, 2006. 